\title{
MicroRNA hsa-miR-623 directly suppresses MMP1 and attenuates IL-8-induced metastasis in pancreatic cancer
}

\author{
YUTONG CHEN $^{1 *}$, SIQI PENG ${ }^{1 *}$, HONG CEN $^{2}$, YUJING LIN $^{3}, \mathrm{CHUMEI} \mathrm{HUANG}^{4}$, YINTING CHEN ${ }^{5,6}$, \\ HONG SHAN $^{7-9}$, YONGHUI SU ${ }^{2}$ and LINJUAN ZENG ${ }^{1}$ \\ ${ }^{1}$ Department of Abdominal Oncology, The Cancer Center of The Fifth Affiliated Hospital of Sun Yat-Sen University; \\ Departments of ${ }^{2}$ General Surgery and ${ }^{3}$ Pathology, The Fifth Affiliated Hospital of Sun Yat-Sen University, Zhuhai, \\ Guangdong 519000; ${ }^{4}$ Department of Gastroenterology, The Seventh Affiliated Hospital of Sun Yat-Sen University, \\ Shenzhen, Guangdong 518107; ${ }^{5}$ Department of Gastroenterology; ${ }^{6}$ Guangdong Provincial Key Laboratory of \\ Malignant Tumor Epigenetics and Gene Regulation, Sun Yat-Sen Memorial Hospital, Sun Yat-Sen University, \\ Guangzhou, Guangdong 510120; ${ }^{7}$ Center for Interventional Medicine; ${ }^{8}$ Guangdong Provincial Key Laboratory of \\ Biomedical Imaging; ${ }^{9}$ Guangdong Provincial Engineering Research Center of Molecular Imaging, \\ The Fifth Affiliated Hospital, Sun Yat-Sen University, Zhuhai, Guangdong 519000, P.R. China
}

Received November 25, 2018; Accepted May 14, 2019

DOI: 10.3892/ijo.2019.4803

\begin{abstract}
Matrix metalloproteinase-1 (MMP1) participates in the metastasis of pancreatic cancer, and its expression can be regulated by endogenous microRNAs (miRs/miRNAs) and exogenous inflammatory factors. Whether miRNAs that potentially modulate MMP1 expression can also attenuate the pro-metastatic effects of its inducer on pancreatic cancer is yet to be completely elucidated. In the present study, a systematic analysis including in silico and bioinformatics analyses, a luciferase reporter assay and an RNA electrophoretic mobility shift assay (EMSA), were used to investigate the
\end{abstract}

Correspondence to: Professor Linjuan Zeng, Department of Abdominal Oncology, The Cancer Center of The Fifth Affiliated Hospital of Sun Yat-Sen University, 52 Mei Hua East Road, Zhuhai, Guangdong 519000, P.R. China

E-mail: zenglinj@mail.sysu.edu.cn

Dr Yonghui Su, Department of General Surgery, The Fifth Affiliated Hospital of Sun Yat-Sen University, 52 Mei Hua East Road, Zhuhai, Guangdong 519000, P.R. China

E-mail:suyh@mail.sysu.edu.cn

*Contributed equally

Abbreviations: ECM, extracellular matrix; EMT-TFs, epithelialmesenchymal transition transcription factors; EMSA, electrophoretic mobility shift assay; FBS, fetal bovine serum; IL-8, interleukin-8; MMP1, matrix metalloproteinase-1; MFE, minimum free energy; miR/miRNA, microRNA; NC, negative control; PDAC, pancreatic ductal adenocarcinoma; PAR1, protease-activated receptor 1; TNF- $\alpha$, tumor necrosis factor- $\alpha$; TCGA, The Cancer Genome Atlas; 3'-UTR, 3'-untranslated region

Key words: pancreatic cancer, hsa-miR-623, metastasis, matrix metalloproteinase-1, IL-8 interaction between miRNAs and MMP1 mRNA. In addition, wound-healing assays, Transwell assays and xenograft nude mouse models were implemented to investigate the antitumor activities exerted by candidate miRNAs. As a result, hsa-miR-623 was screened as a candidate miRNA that interacts with the MMP1 transcript, and an inverse correlation between the expression of hsa-miR-623 and MMP1 was observed in human pancreatic cancer tissue samples. The EMSA confirmed that hsa-miR-623 was able to directly bind to its cognate target within the 3'-untranslated region of the MMP1 transcript. In addition, transfection of hsa-miR-623 mimics into PANC-1 and BXPC-3 cell lines markedly inhibited the expression of MMP1 at the mRNA and protein levels, and attenuated IL-8-induced MMP1 expression. hsa-miR-623 also decreased IL-8-induced epithelial-mesenchymal transition in PANC-1 and BXPC-3 cells via the underlying mechanism of inhibition of ERK phosphorylation. Consequently, hsa-miR-623 inhibited pancreatic cancer cell migration and invasion in vitro and metastasis in vivo. The results of the present study suggest that hsa-miR-623 represents a novel adjuvant therapeutic target to prevent metastasis in pancreatic cancer.

\section{Introduction}

Pancreatic cancer is one of the most lethal malignancies worldwide. Numerous factors, such as difficulty in establishing an early diagnosis, recurrence, metastasis and resistance to chemotherapy and radiotherapy, contribute to the poor prognosis of this disease. Over the past 30 years, the long-term survival rate of patients with pancreatic cancer has remained low (as low as 6\%) (1). A high rate of metastasis is one of the principal causes of therapy failure in patients with pancreatic cancer according to an autopsy series, which reported that distant metastasis occurs in $90 \%$ of cases (2). As the benefits from surgical resection are limited for patients with early recurrence or distant metastasis, and as chemotherapy, 
radiation therapy and palliative care are insufficient to prevent metastasis, the identification of novel therapeutic methods or targets for pancreatic cancer is urgently required (3).

Gradients of the extracellular matrix (ECM) serve important roles in supporting tumor cell proliferation, adhesion, migration and survival (4). Matrix metalloproteinase-1 (MMP1) degrades the ECM and basement membrane components, resulting in tumor progression via the remodeling of the ECM. Several factors contribute to the expression of MMP1, including endogenous factors, such as polymorphisms and epigenetic regulation in the promoter region of MMP1, as well as exogenous factors, such as the tumor microenvironment. A variety of MMP1 promoter genotypic polymorphisms have been reported in various ethnic populations, and their contributions to different disease risks have been explored (5-7). In the tumor microenvironment, a variety of inflammatory factors, including interleukin-8 (IL-8) (8), IL-1 $\beta$ (9) and tumor necrosis factor- $\alpha$ (TNF- $\alpha)(10)$, have been reported to induce MMP1 expression in cancer cells.

Another important mechanism that influences the expression of MMP1 is epigenetic modification. MicroRNAs (miRs/miRNAs) are a group of small non-coding RNAs that have been identified as important regulators of MMP gene expression at the post-transcriptional level (11). miRNA-mediated MMP regulation is considered to be a key factor involved in neoplastic tumors, and is closely associated with the clinicopathological features and outcomes of patients (12). miRNAs may serve as promising biomarkers for predicting the therapeutic response or for prognostic evaluation, and may additionally serve as potential therapeutic targets for various types of cancer, including pancreatic cancer (13-15). Several miRNAs have been identified to target MMP1, such as miR-222 in hypertrophic scars (16), miR-526b in the skin (17), miR-let-7b in stem cells from apical papilla (18) and miR-202-3p in scleroderma fibrosis (19). However, whether the miRNA modulation of MMP1 expression could attenuate metastasis induced by inflammatory factors in pancreatic cancer requires further investigation, as it is yet to be completely elucidated.

In the present study, a systematical analysis, combining in silico, in vitro, in vivo and bioinformatics approaches, was performed to investigate the interactions between miRNAs and MMP1. miRNA-623 was observed to suppress MMP1 by targeting its 3'-untranslated region (3'-UTR), attenuate IL-8-induced MMP1 expression, and inhibit pancreatic cancer cell migration, invasion and metastasis in vitro and in vivo.

\section{Materials and methods}

Cell lines. The 293T and human pancreatic cancer PANC-1 and BXPC-3 cell lines were purchased from the American Type Culture Collection and were incubated at $37^{\circ} \mathrm{C}$ in a humidified incubator containing $5 \% \mathrm{CO}_{2}$. The cells were cultured in DMEM supplemented with $10 \%$ fetal bovine serum (FBS) (both from Thermo Fisher Scientific, Inc.), $100 \mathrm{U} / \mathrm{ml}$ penicillin and $100 \mu \mathrm{g} / \mathrm{ml}$ streptomycin (both Jiangsu KeyGEN BioTECH Corp., Ltd.).

Mice.Atotalof20femalenudemice(BALB/cJNju-Foxn1nu/Nju; age, 5 weeks; weight, $16 \pm 2.5 \mathrm{~g}$ ) were purchased from Nanjing Biomedical Research Institute (http://www.nbri-nju. com/en-us/about-animal-facility). All the animals were raised under pathogen-free conditions at $20-26^{\circ} \mathrm{C}, 40-70 \%$ relative humidity, 15 times/h ventilation and a $12 \mathrm{~h}$ light/12 h dark cycle. The animals were provided with water and food freely. All animal experiments were approved by the Animal Care and Use Committee of Sun Yat-Sen University (Guangzhou, China).

Reagents and chemicals. Synthetic miR-623 mimics (5'-ACC CAACAGCCCCUGCAAGGGAU-3'), inhibitors (5'-CAGUAC UUUUGUGUAGUACAA-3'), miR-623 mutant (5'-ATAACTTGAAGGGGCTGTTGGGT-3'), mimics negative control (NC; 5'-UUCUCCGAACGUGUCACGUTTACGUG ACACGUUCGGAGAATT-3') and inhibitor NC (5'-CAGUACU UUUGUGUAGUACAA-3') miRNAs were purchased from Shanghai GenePharma Co., Ltd. Recombinant human IL-8 (rIL-8) was purchased from PeproTech, Inc. Rabbit-anti-human MMP1 (cat. no. 54376), ERK (cat. no. 4695), phosphorylated (p-)ERK (cat. no. 4370), Twist-related protein 1 (TWIST1; cat. no. 46702), zinc finger protein SNAI1 (cat. no. 3879) and E-cadherin (cat. no. 14472) polyclonal primary antibodies, mouse GAPDH (cat. no. 51332) monoclonal primary antibody and horseradish peroxidase-conjugated secondary antibodies (anti-rat cat. no. 7077; anti-rabbit cat. no. 7074) were purchased from Cell Signaling Technology, Inc. Phalloidin was purchased from Sigma-Aldrich; Merck KGaA, and DAPI was from Wuhan Boster Biological Technology, Ltd.

In silico analyses. The microRNA.org (http://www.microrna. org/), PITA (http://genie.weizmann.ac.il/pubs/mir07/mir07_ prediction.html), TargetScan (Release 6.2, http://www. targetscan.org) and miRTar.human (http://mirtar.mbc.nctu. edu.tw/human/) databases were screened for potential miRNAs binding to the 3'-UTR of the MMP1 transcript (acc. no. NM_002421). The minimum free energy (MFE) of hybridization was calculated using the RNAhybrid program (http://bibiserv2.cebitec.uni-bielefeld.de/rnahybrid).

Luciferase reporter assay. The pGL3-Control vector (Promega Corporation) and the pGL3-CU vector were constructed according to the protocol outlined in previous studies $(20,21)$. The sequence of the MMP1 3'-UTR was amplified via PCR using primers listed in Table I. The PCR products were digested with USER enzyme (New England BioLabs, Inc.) and ligated into the pGL3-CU vector, resulting in a pGL3-MMP1 vector, according to the manufacturer's protocols. The authenticity of pGL3-MMP1 vector was confirmed.

To verify the precise target of the miRNAs, the Dual-Luciferase Reporter Assay system (Promega Corporation) was used. Briefly, 293T cells were seeded in 96-well plates at a density of $1 \times 10^{5}$ cells per well and cultured in complete medium without antibiotics for $24 \mathrm{~h}$. The luciferase reporter plasmids pGL3-NC (Promega Corporation) and pGL3-MMP1 (100 ng/well) were then co-transfected with a mimic NC, hsa-miR-623 mimic, inhibitor NC, hsa-miR-623 inhibitor or hsa-miR-623 mutant (50 nM) using Lipofectamine $^{\circledR} 3000$ (Invitrogen; Thermo Fisher Scientific, Inc.). Firefly and Renilla luciferase activities were measured at $24 \mathrm{~h}$ post-transfection using the Dual-Luciferase Reporter Assay kit. Firefly luciferase was normalized to Renilla 
Table I. Sequences of primers and oligonucleotides used in this study.

\begin{tabular}{lll}
\hline Assay & \multicolumn{1}{c}{ Name } & \multicolumn{1}{c}{ Sequence $\left(5^{\prime}-3^{\prime}\right)$} \\
\hline Luciferase reporter gene assay & MMP1 3'-UTR forward & CCAAAGAAGGTGTTTCCTG \\
& MMP1 3'-UTR reverse & GAAAGTGTATAAAACAGTAG \\
hsa-miR-623 mutant & ATAACTTGAAGGGGCTGTTGGGT \\
Electrophoretic & dye-miR-623 & /5IRD800CWN/rArUrCrCrCrUrUrGrCrArGrGrGrGrCrUrGrUr \\
mobility shift assay & UrGrGrGrU \\
& cold-miR-623 & /rArUrCrCrCrUrUrGrCrArGrGrGrGrCrUrGrUrUrGrGrGrU \\
& dye-miR-623-TAR & /5Cy55/mUmCmAmCmCmCmUmGmGmAmUmAmGmGm \\
& Cold-negative control & /rUrCrArCrArArCrCrUrCrCrUrArGrArArArGrArGrUrArGrA \\
Quantitative PCR & AMP1 forward & ACACGCCAGATTTGCCAAGAGC \\
& GMP1 reverse & GGAGAGTTGT CCCGATGATCTCCCC \\
& GAPDH forward & GAATCCCATCACCATCTTCCAGG \\
& GAPDH reverse & GAGCCCCAGCCTTCTCCATG \\
hsa-miR-623 forward & ATCCCTTGCAGGGGCTGTTGGGT \\
U6 forward & CTCGCTTCGGCAGCACA \\
miR universal reverse & AACGCTTCACGAATTTGCGT
\end{tabular}

miR, microRNA; MMP1, matrix metalloproteinase-1; 3'-UTR, 3'-untranslated region.

luciferase activity. Three independent experiments were performed.

RNA electrophoretic mobility shift assay (EMSA). An RNA EMSA was performed using a LightShift Chemiluminescent RNA EMSA kit (Thermo Fisher Scientific, Inc.) according to the manufacturer's protocol. The binding buffer mixture was prepared with 1X REMSA binding buffer supplemented with $5 \%$ glycerol, $200 \mathrm{mM} \mathrm{KCl}$ and $100 \mathrm{mM} \mathrm{MgCl}_{2}$. Next, $200 \mathrm{nmol}$ IRDye1800-labeled hsa-miR-623 and/or Cy5.5-labeled hsa-miR-623 TAR (targeting sequences in the MMP1 3-'UTR) oligonucleotides (Integrated DNA Technologies, Inc.) were added to the binding buffer mixture. Cytoplasmic protein from PANC-1 cells was extracted using NE-PER Nuclear and Cytoplasmic Extraction reagents (Thermo Fisher Scientific, Inc.). The oligonucleotide sequences are listed in Table I.

To confirm the binding specificity between hsa-miR-623 and its target, 50-fold molar excess of cold-hsa-miR-623 or cold-NC probe was added to the reaction system $5 \mathrm{~min}$ prior to the addition of the dye-labeled probes. A total of $2 \mu \mathrm{g}$ PANC- 1 cell cytoplasmic protein and $1 \mu \mathrm{g}$ tRNA were added to the hsa-miR-623/target mixtures to examine the RNA-protein interactions. Following a 20-min incubation at room temperature, the reaction mixture was loaded onto a $10 \%$ gel for PAGE and separated by electrophoresis $(100 \mathrm{~V} ; 150 \mathrm{~min})$ at $4^{\circ} \mathrm{C}$. Finally, the bands were detected using an Odyssey CLx Infrared Imaging system (LI-COR Biosciences).

Incubation with rIL-8. PANC-1 and BXPC-3 cells were seeded at a density of $6 \times 10^{5}$ cells per well in 6-well plates and cultured for $12 \mathrm{~h}$ to allow cells to fully attach. The medium was then replaced with serum-free medium, and rIL- 8 was added to a final concentration of $100 \mathrm{ng} / \mathrm{ml}(22,23)$, followed by incubation for $6,12,24,36$ and $48 \mathrm{~h}$. The cells were then harvested for further analyses.
Cell transfection. Synthetic miR-623 mimics, inhibitors and NC miRNAs were transfected into PANC-1 and BXPC-3 cells using Lipofectamine 2000 reagent (Invitrogen; Thermo Fisher Scientific, Inc.). PANC-1 and BXPC-3 cells were seeded at a density of $6 \times 10^{5}$ cells per well in 6 -well plates, $3 \mu$ t transfection reagent was added into each well, and the final concentration of microRNA (mimic NC, mimic, inhibitor NC or inhibitor) was $20 \mathrm{nmol} / \mathrm{l}$ in a $2-\mathrm{ml}$ final volume. At $6 \mathrm{~h}$ following incubation, the medium was changed to fresh complete medium, and the cells were harvested $48 \mathrm{~h}$ post-transfection. The transfection efficiency was determined by reverse transcription-quantitative PCR (RT-qPCR).

For stable transfections, $3 \mu \mathrm{g}$ miR-623-GFP or NC-GFP vector were packaged into lentiviral virus vectors, according to the manufacturer's protocol (Vigene Biosciences, Inc.). The PANC-1 cells were then transfected with the lentiviruses and selected with $2 \mu \mathrm{g} / \mathrm{ml}$ puromycin (Sigma-Aldrich; Merck KGaA). The transfection efficiency was evaluated by RT-qPCR.

$R T-q P C R$. Total RNA was extracted from PANC-1 and BXPC-3 cells using the miRNeasy Mini kit (Qiagen $\mathrm{GmbH}$ ). The RNA concentration and purity were determined according to the A260/280 values, using a NanoDrop spectrophotometer (NanoDrop Technologies; Thermo Fisher Scientific, Inc.). A High-Capacity cDNA Reverse Transcription kit (Applied Biosystems; Thermo Fisher Scientific, Inc.) or miScript ${ }^{\circledR}$ II RT kit (Qiagen $\mathrm{GmbH}$ ) was used for first-strand cDNA synthesis. The samples were reverse transcribed at $37^{\circ} \mathrm{C}$ for $15 \mathrm{~min}$ and heated to $85^{\circ} \mathrm{C}$ for $5 \mathrm{sec}$ to inactivate the reverse transcriptase.

The qPCR was performed on an ABI Prism 7900 Sequence Detection system (Applied Biosystems; Thermo Fisher Scientific, Inc.) using a QuantiFast SYBR1 Green RT-PCR kit (Qiagen $\mathrm{GmbH}$ ). The reactions were preheated for $2 \mathrm{~min}$ at 
$50^{\circ} \mathrm{C}$ and denatured for $2 \min$ at $90^{\circ} \mathrm{C}$, followed by 40 cycles of denaturation at $95^{\circ} \mathrm{C}$ for $15 \mathrm{sec}$ and annealing/extension at $60^{\circ} \mathrm{C}$ for $15 \mathrm{sec}$. The $\mathrm{qPCR}$ results, recorded as threshold cycle numbers $(\mathrm{Ct})$, were calculated by the $2^{-\Delta \Delta \mathrm{Cq}}$ method with normalization against GAPDH mRNA as an internal control (24). The relative expression levels of MMP1 and hsa-miR-623 were normalized to the housekeeping gene GAPDH and U6 small nuclear RNA, respectively. The primers used in the RT-qPCR were obtained from Integrated DNA Technologies, Inc. and their sequences are listed in Table I.

Western blot analysis. Protein extracts from PANC-1 and BXPC-3 cells were prepared using RIPA lysis buffer with protease inhibitors and quantified using a bicinchoninic acid protein assay kit (Pierce; Thermo Fisher Scientific, Inc.). A total of $20 \mu \mathrm{g}$ protein from each experimental group was separated on a polyacrylamide gel containing $10 \%$ sodium dodecyl sulfate and subsequently transferred to a $0.45-\mu \mathrm{m}$ polyvinylidene difluoride membrane (EMD Millipore). The membrane was then blocked with $5 \%$ non-fat milk at room temperature for $1 \mathrm{~h}$. Following an incubation with the primary polyclonal primary antibodies MMP1 (1:1,000 dilution), TWIST1 (1:500 dilution), SNAI1 (1:1,000 dilution), E-cadherin (1:400 dilution), ERK (1:1,000 dilution), p-ERK (1:2,000 dilution) or GAPDH (1:2,000 dilution) overnight at $4^{\circ} \mathrm{C}$ and incubation with the secondary antibodies (1:2,000 dilution) for $1 \mathrm{~h}$ at room temperature, protein bands were detected using enhanced chemiluminescence reagents (Thermo Fisher Scientific, Inc.) and visualized after exposure using the Tanon-5200 Multi-imaging system (Tanon Science and Technology Co., Ltd.). ImageJ version 1.8.0 software (National Institutes of Health) was used to analyze the images.

Immunofluorescence assay. Following transfection with synthetic miR-623 mimics, inhibitors and NC miRNAs, and co-culturing with IL-8, PANC-1 and BXPC-3 cells were fixed with $4 \%$ formaldehyde for $20 \mathrm{~min}$ at room temperature. The cells were then stained with $5 \mu \mathrm{g} / \mathrm{ml}$ phalloidin for $30 \mathrm{~min}$, counterstained with $1 \mu \mathrm{g} / \mathrm{ml}$ DAPI for $10 \mathrm{~min}$ at room temperature, and finally visualized using a fluorescence scanning microscope at x400 magnification.

Wound-healing assay. PANC-1 and BXPC-3 cells were transfected with synthetic miR-623 mimics, inhibitors and $\mathrm{NC}$ miRNAs, and a wound-healing assay was performed $48 \mathrm{~h}$ after transfection. When the cells reached $100 \%$ confluence, a wound was created by manually scraping the cell monolayer with a $200-\mu \mathrm{l}$ pipette tip. At 0 and $24 \mathrm{~h}$ after incubation, images were captured using a light microscope (Zeiss $\mathrm{GmbH}$ ) at x40 magnification, and analyzed quantitatively by ImageJ version 1.8.0 software.

Transwell invasion assay. An invasion assay was performed using $8-\mu \mathrm{m}$ pore, 24 -well Transwell chambers coated with Matrigel (Corning Life Sciences). PANC-1 and BXPC-3 cells were transfected with synthetic miR-623 mimics, inhibitors and NC miRNAs. At $48 \mathrm{~h}$ after transfection, the cells were harvested, $5 \times 10^{4}$ cells in $200 \mu \mathrm{l}$ serum-free medium were reseeded into the top chamber, and the bottom chamber was filled with $750 \mu 1$ DMEM containing 10\% FBS. Cells that migrated to the underside of the membrane were stained with $0.1 \%$ crystal violet (Phygene Life Sciences Co., Ltd.) for $30 \mathrm{~min}$ at room temperature, followed by imaging and counting under a light microscope (Zeiss $\mathrm{GmbH}$ ) at x40 magnification. All experiments were performed in triplicate and repeated $\geq 3$ times.

In vivo efficacy studies. To investigate the effect of hsa-miR-623 on the metastasis of pancreatic cancer cells, $1 \times 10^{6}$ PANC-1 cells stably expressing miR-623 or NC were injected into the tail veins of mice, which were divided into 4 groups $(n=5)$ : PANC-1 ${ }^{\text {Lenti-GFP }}$, PANC-1 $1^{\text {Lenti-miR-623-GFP }}$, PANC-1 ${ }^{\text {Lenti-GFP+IL-8 }}$ and PANC-1 ${ }^{\text {Lenti-miR623-GFP+IL-8 }}$. The rIL-8 was administered via intraperitoneal injection at $100 \mathrm{ng} /$ animal once a week for 5 weeks. The weight, activity and appearance of the mice were monitored every 3 days. If the body weight of the animal dropped markedly in a short time, the subcutaneous fat disappeared, the animals exhibited spinal curvature, and the activity and reaction ability decreased, euthanasia was carried out in advance, otherwise euthanasia was carried out at 5 weeks. The mice were anaesthetized by intraperitoneal injection of $0.5 \%$ pentobarbital sodium salt (CAS, 57-33-0; Beijing Huayehuanyu Chemical Co., Ltd.) at a dosage of $100 \mathrm{mg} / \mathrm{kg}$, and then placed in transparent glass containers. $100 \%$ carbon dioxide was introduced into the bottles with the filling rate of $10-30 \%$ volume per min. The death of the mice was confirmed when no spontaneous breathing was detected for 2-3 min and they exhibited no blinking reflex. Subsequently, the livers and lungs of the animals were dissected and imaged under a fluorescence scanning microscope. All dissected organs were fixed overnight with $4 \%$ formalin at room temperature and then embedded in paraffin. The livers and lungs were sliced to $5-\mu \mathrm{m}$ thickness and stained with hematoxylin for $15 \mathrm{~min}$ and eosin for $1 \mathrm{~min}$ at room temperature. Immunochemical assays were performed according to the protocols of the immunohistochemical staining kit manufacturer (cat. no. SA1022; Wuhan Boster Biological Technology, Ltd.), using the aforementioned primary antibodies incubated overnight at $4^{\circ} \mathrm{C}$. The numbers of liver and lung metastases were independently calculated and assessed by two pathologists.

The Cancer Genome Atlas (TCGA) and Gene Expression Omnibus (GEO) dataset analysis. The clinicopathological characteristics, mRNA expression levels of MMP1 and IL-8, and the hsa-miR-623 levels in pancreatic ductal adenocarcinoma (PDAC) tissues were obtained from TCGA database (https://cancergenome.nih.gov/), which contained 172 patients with PDAC. As there are only 4 normal samples included in TCGA database, the present study also searched other GEO (https://www.ncbi.nlm.nih.gov/geo/) public datasets for further data, including datasets GES28735 (45 paired PDAC and adjacent non-tumor tissues), GSE32678 and GSE32676 (data for miRNA and mRNA expression levels in 25 PDAC tissues) $(25,26)$.

Statistical analysis. All statistical analyses were performed using SPSS version 17.0 software (SPSS, Inc.). The results are presented as the mean \pm standard deviation. Pearson's correlation analysis was used to analyze the correlation between MMP1 mRNA levels and hsa-miR-623 levels, and 
between MMP1 mRNA levels and IL-8 mRNA levels in the original data from human PDAC tissues published in the GEO or TCGA databases. One-way analysis of variance was used to compare data from multiple groups, and the Bonferroni method was used to determine the significant differences between the groups. $\mathrm{P}<0.05$ was considered to indicate a statistically significant difference.

\section{Results}

Identification of potential miRNAs that regulate MMPl. The screening results from all four databases suggested that several miRNAs may target the 3'-UTR of the MMP1 transcript. The correlation between the expression of miRNA and the expression of MMP1 in human PDAC tissues was analyzed, and the results are presented in Table II. According to our previous studies, the value of MFE $\leq-20 \mathrm{kcal} / \mathrm{mol}$ was considered to be essential for the miRNA/mRNA hybridization $(20,21,27-29)$. As presented in Table II, the MFE of hybridization between hsa-miR-623 and its cognate target at the 3'-UTR of MMP1 was $-27 \mathrm{kcal} / \mathrm{mol}$. Based on the database analysis, compared with adjacent non-tumor tissues, the expression of MMP1 in PDAC tumor tissues was increased, whereas the expression of hsa-miR-623 was decreased, and a negative correlation was observed between their expression $(r=-0.402$, $\mathrm{P}=0.046$; Fig. 1A). Based on these results, hsa-miR-623 was identified as a candidate miRNA for further investigation.

hsa-miR-623 directly interacts with its target within the MMPI 3 '-UTR. RNA EMSA was performed to explore whether direct binding occurs between hsa-miR-623 and its target within the MMP1 3'-UTR. As illustrated in Fig. 1B, complexes formed between hsa-miR-623 and their cognate MMP1 mRNA target sequences in vitro (lanes 3-5, the duplex is indicated by an arrowhead). Competition assays indicated that the complex of hsa-miR-623 and its cognate targets was sequence-specific, as the complex formation was inhibited by a 50 -fold molar excess of cold hsa-miR-623 (lane 5); however, was not influenced by a 50-fold molar excess of unlabeled NC oligonucleotide (lane 4). PANC-1 cytoplasmic extracts were subsequently used to observe the formation of miRNA/mRNA/protein complexes (lanes 6-8, complexes A and B). Taken together, the competition assays revealed that complex A was inhibited by a 50 -fold molar excess of cold hsa-miR-623 (lane 8), suggesting that hsa-miR-623 directly and specifically interacts with its target within the MMP1 3'-UTR.

hsa-miR-623 suppresses MMP1 3'-UTR luciferase reporter gene activity, MMP1 endogenous expression, and the migration and invasion of pancreatic cancer cells. Luciferase reporter assays were performed to explore whether hsa-miR-623 could regulate the 3'-UTR of MMP1. The mutant hsa-miR-623 was designed with 3 cytosines replaced by adenines; consequently, the free energy of hybridization between the mutated miRNA and its target mRNA changed from -27 to $-16.8 \mathrm{kcal} / \mathrm{mol}$ (Fig. 1C). Following dual transfection, the luciferase activity produced by the reporter gene plasmid was significantly downregulated by the wild-type hsa-miR-623 mimic $(0.64 \pm 0.11 ; \mathrm{P}<0.05)$ and increased by the hsa-miR-623 inhibitor $(1.52 \pm 0.15 ; \mathrm{P}<0.05)$. Conversely, the
Table II. Candidate miRs targeting the 3'-untranslated region of MMP1 and expression correlation in pancreatic ductal adenocarcinoma tissues ${ }^{\mathrm{a}}$.

\begin{tabular}{lcccc}
\hline \multicolumn{5}{c}{ Correlation } \\
miR name & $\mathrm{R}$ & P-value & kcal/mol & Position $^{\mathrm{c}}$ \\
\cline { 2 - 5 } hsa-miR-623 & -0.4025 & 0.046 & -27.0 & $8158-8179$ \\
hsa-miR-127-5p & -0.293 & 0.155 & -22.0 & $8046-8063$ \\
hsa-miR-188-5p & 0.050 & 0.811 & -28.2 & $8159-8180$ \\
hsa-miR-190b & -0.2661 & 0.199 & -21.6 & $8057-8077$ \\
hsa-miR-1915-5p & -0.1671 & 0.425 & -27.8 & $8159-8177$ \\
hsa-miR-330-5p & 0.0296 & 0.888 & -24.2 & $8032-8053$ \\
hsa-miR-361-5p & -0.030 & 0.888 & -24.0 & $7838-7859$ \\
hsa-miR-526a & -0.223 & 0.283 & -20.6 & $7828-7849$ \\
hsa-miR-518c-5p & -0.030 & 0.888 & -19.0 & $8006-8026$ \\
hsa-miR-518f-5p & -0.308 & 0.134 & -19.6 & $7828-7849$ \\
hsa-miR-519a-5p & -0.337 & 0.099 & -20.6 & $7828-7849$ \\
hsa-miR-518e-5p & -0.101 & 0.631 & -20.6 & $7828-7849$ \\
hsa-miR-523-5p & -0.337 & 0.099 & -20.6 & $7828-7849$ \\
hsa-miR-558 & 0.177 & 0.397 & -23.3 & $8115-8133$ \\
hsa-miR-769-3p & 0.237 & 0.253 & -24.4 & $8029-8050$ \\
hsa-miR-638 & -0.104 & 0.620 & -26.3 & $8028-8049$ \\
hsa-miR-936 & -0.173 & 0.408 & -20.5 & $8220-8241$ \\
& & & & \\
\hline
\end{tabular}

${ }^{a}$ mRNA and microRNA data were obtained from datasets GSE32676 and GSE32678, respectively. ${ }^{\mathrm{b} C}$ Calculated using the RNAhybrid program (http://bibiserv2.cebitec.uni-bielefeld.de/rnahybrid). ${ }^{\circ}$ The nucleotide position at the 3'-UTR of MMP1 (acc. no. NM_002421), where the first nucleotide at the transcription start site is counted as position 1 . miR, microRNA; 3'-UTR, 3'-untranslated region; MMP1, matrix metalloproteinase- 1 .

mutant hsa-miR-623 mimic failed to influence the luciferase activity in the $293 \mathrm{~T}$ cells (Fig. 1D). These results indicate that hsa-miR-623 modulates its target in the 3'-UTR of MMP1 in a direct and specific manner. Following synthetic miRNA transfection, mimics increased and inhibitors decreased hsa-miR-623 expression levels in the PANC-1 and BXPC-3 cells (Fig. 2A and D). Consistent with the results from the EMSA and luciferase assay, the hsa-miR-623 mimic suppressed endogenous MMP1 expression at the mRNA and protein levels, whereas the hsa-miR-623 inhibitor led to an increase in MMP1 expression (Fig. 2B, C, E and F). Furthermore, the hsa-miR-623 mimic resulted in a decrease in the migration and invasion ability of PANC-1 and BXPC-3 cells, whereas the hsa-miR-623 inhibitor displayed the opposite effects (all $\mathrm{P}<0.05$; Fig. 3A and $\mathrm{B}$ ).

hsa-miR-623 attenuates IL-8-induced MMP1 expression, and the migration and invasion of pancreatic cancer cells. In rheumatoid arthritis, MMP1 can be induced by TNF- $\alpha$ and IL-1 $\beta(30,31)$, however, whether MMP1 can be induced by inflammatory factors in PDAC cells remains unclear. First, the correlation was investigated between the expression of MMP1 mRNA and 3 commonly studied inflammatory factors, IL-1 $\beta$, IL- 6 and IL-8. The results based on TCGA 

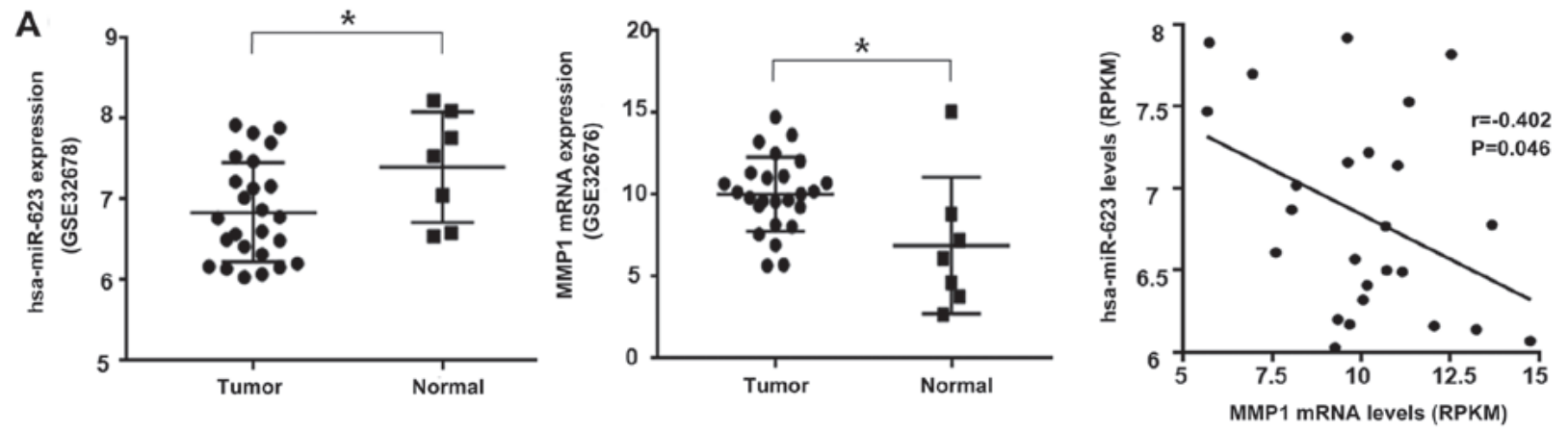

B miR-623 mimic
mRNA
Cold NC
Cold miR-623 mimic
tRNA
Cell protein extraction
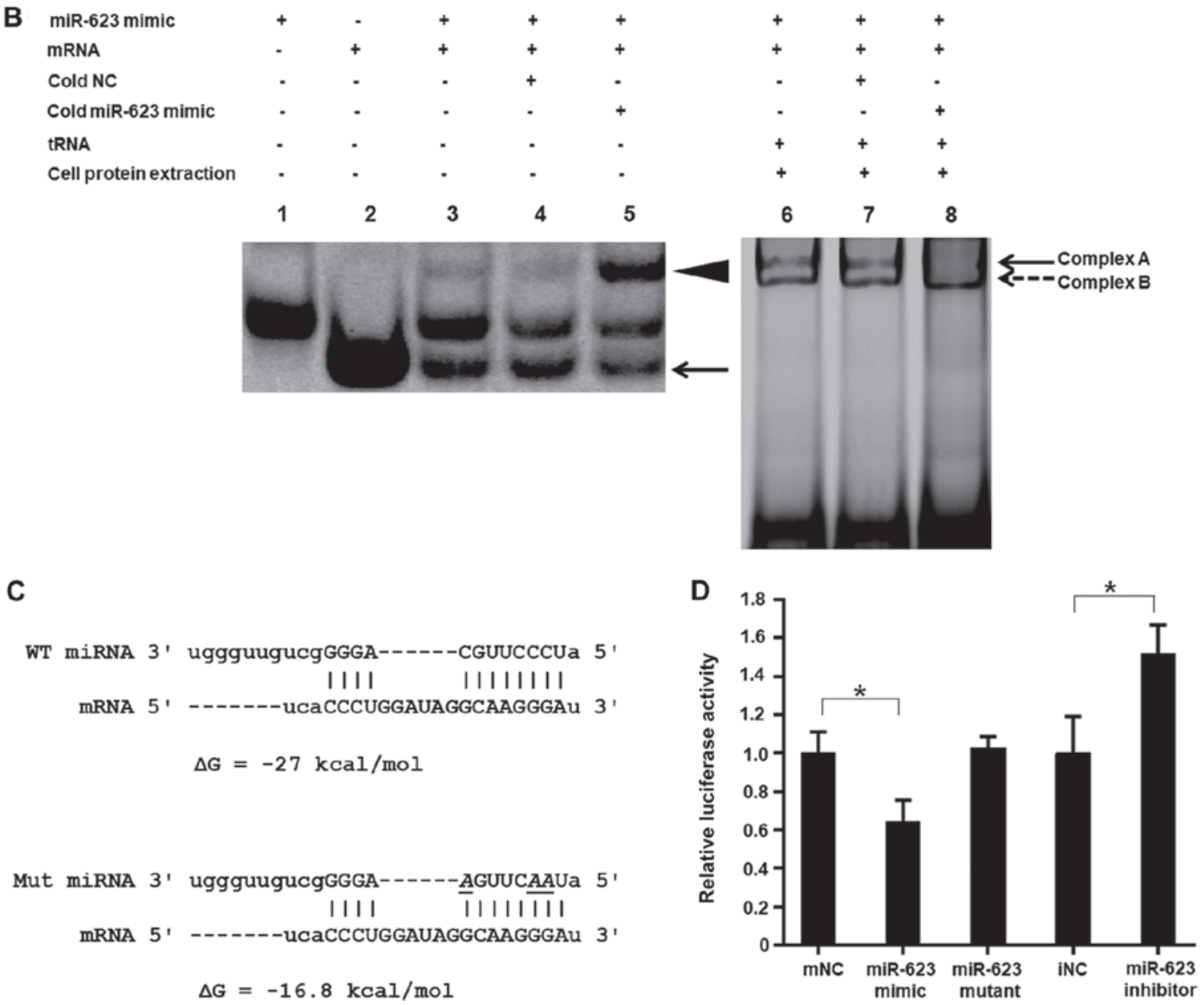

Figure 1. hsa-miRNA-623 modulates MMP1 by targeting its 3'-UTR. (A) The expression of MMP1 in PDAC tumor tissues was increased; however, the expression of hsa-miR-623 was decreased compared with adjacent non-tumor tissues, and the levels of hsa-miR-623 were negatively correlated with MMP1 mRNA levels in human PDAC tissues (datasets GSE32678 and GSE32676). ${ }^{*} \mathrm{P}<0.05$. (B) RNA electrophoretic mobility shift assays. In lane 4 the cold NC did not affect hybridization, and in lane 5 hybridization was inhibited, as most of the mRNA was bound by cold hsa-miR-623. The arrowhead indicates miR/mRNA hybridization, and the arrow indicates free mRNA. miR/mRNA/protein complex formation is evident in lanes 6-8 (complexes A and B) and was not affected by cold NC, yet was inhibited by a 50-fold molar excess of cold hsa-miR-623 (lane 8). (C) In silico analyses of the interaction between hsa-miRNA-623 and its target sequence within the 3'-UTR of MMP1 mRNA. The free energy of the predicted hybrid complex formed by hsa-miR-623 and its target was $-27 \mathrm{kcal} / \mathrm{mol}$. When 3 nucleotides in the hsa-miR-623 sequence were altered (italic, underlined), the predicted free energy of hybridization changed to $-16.8 \mathrm{kcal} / \mathrm{mol}$. (D) hsa-miR-623 specifically regulated luciferase activity produced by the reporter gene plasmid containing the 3'-UTR of MMP1 in 293T cells, whereas mutant hsa-miR-623 did not have a significant influence on luciferase activity. The data are presented as the mean \pm standard deviation $(\mathrm{n}=3)$. " $\mathrm{P}<0.05$. miR, microRNA; MMP1, matrix metalloproteinase-1; 3'-UTR, 3'-untranslated region; RPKM, Reads Per Kilobase Million; NC, negative control; mNC, mimic NC; iNC, inhibitor NC.

database (Fig. 4A-C) revealed that the correlation between IL-8 and MMP1 ( $\mathrm{r}=0.445)$ was higher than that with IL-1 $\beta$ $(\mathrm{r}=0.257)$, and both were statistically significant. However, only IL-8 exhibited significant correlation with MMP1 expression in the GEO dataset GSE28735 (Fig. 4D, E and F). Based on the results of the analysis from the two databases, IL- 8 was selected as an inducer of MMP1. As presented in Fig. 5A, at 24, 36 and $48 \mathrm{~h}$ of co-culturing with rIL-8, the expression of MMP1 was significantly upregulated (all $\mathrm{P}<0.05$ ). When the hsa-miR-623 mimic was transfected into PANC-1 and BXPC-3 cells prior to 
A

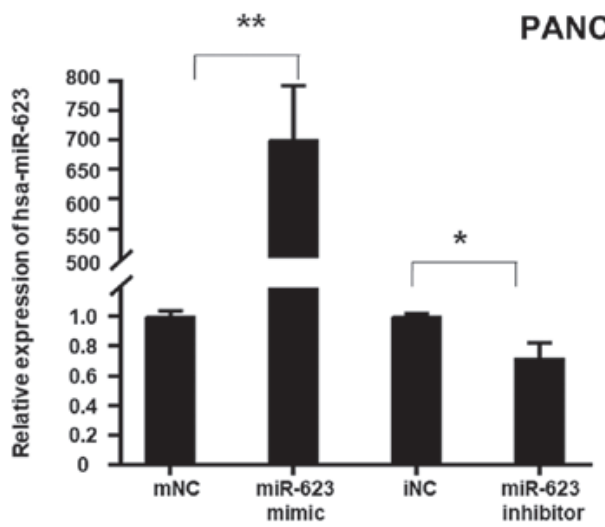

C

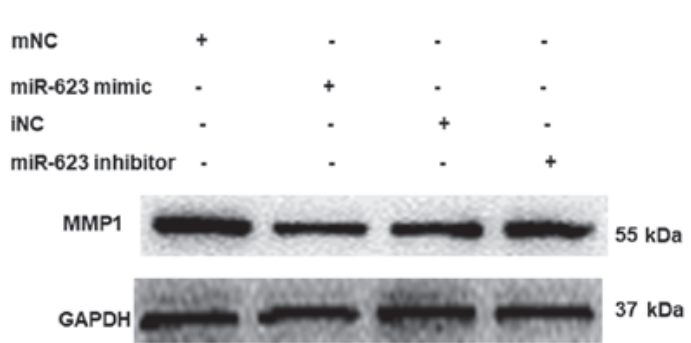

D

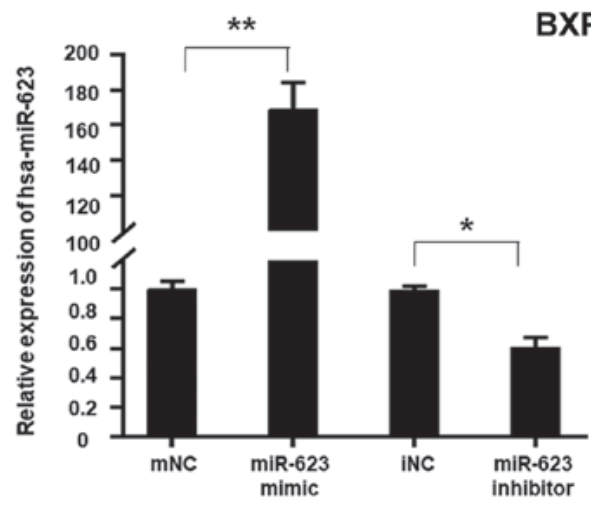

$\mathbf{F}$

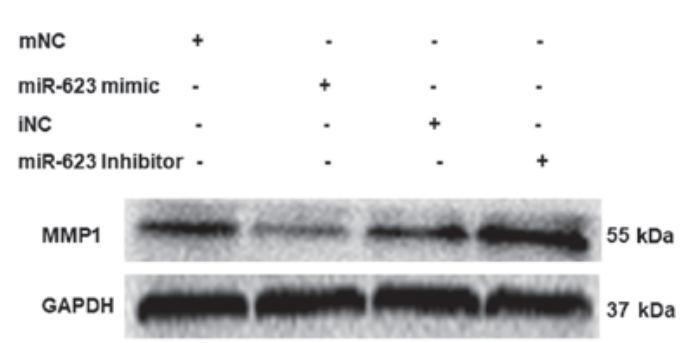

B
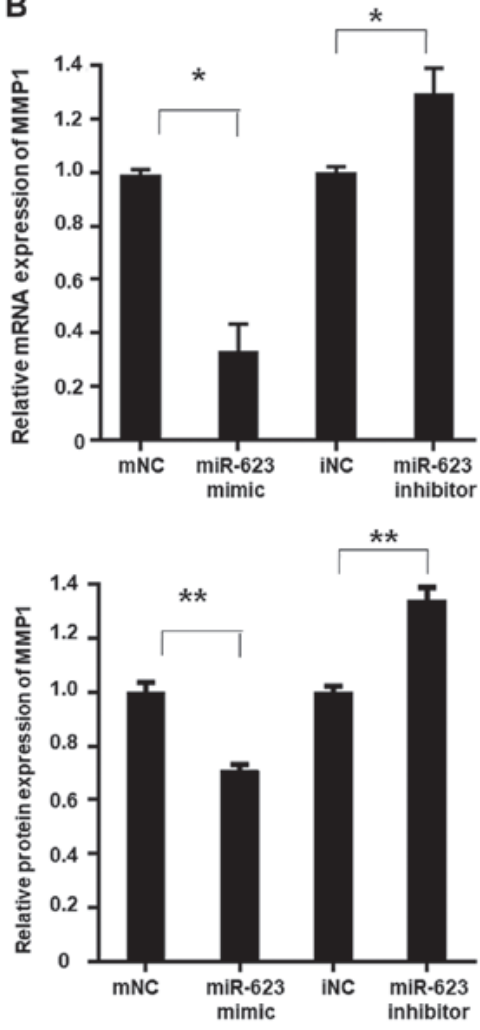

E
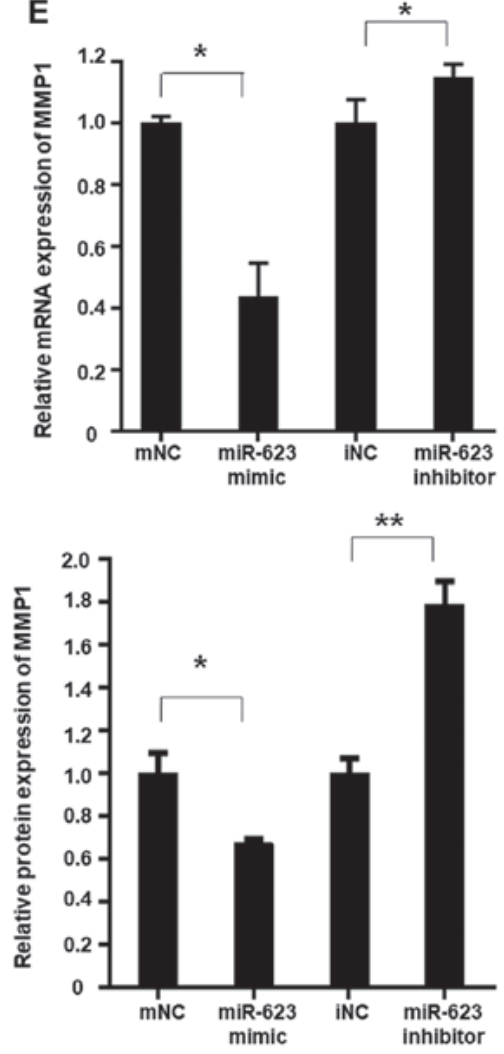

Figure 2. hsa-miR-623 suppresses endogenous MMP1 expression in PANC-1 and BXPC-3 cells. Cells were transiently transfected using 50 nmol/1 hsa-miR-623 mimic, mNC, hsa-miR-623 inhibitor or iNC. Transfection of the hsa-miR-623 mimic significantly increased hsa-miR-623 levels in (A) PANC-1 and (D) BXPC-3 cells, whereas the hsa-miR-623 inhibitor decreased the hsa-miR-623 levels. The hsa-miR-623 mimic suppressed, whereas the hsa-miR-623 inhibitor upregulated, endogenous MMP1 expression in (B and C) PANC-1 and (E and F) BXPC-3 cells. The data are presented as the mean \pm standard deviation $(\mathrm{n}=3) .{ }^{*} \mathrm{P}<0.05,{ }^{* *} \mathrm{P}<0.001$. miR, microRNA; MMP1, matrix metalloproteinase-1; mNC, mimic negative control; iNC, inhibitor negative control.

treatment with rIL-8, the induction of MMP1 was attenuated, whereas the hsa-miR-623 inhibitor transfection further increased the MMP1 expression (Fig. 5B). In addition, the expressions of TWIST1 and SNAI1 were increased following IL-8 treatment 
A

PANC-1
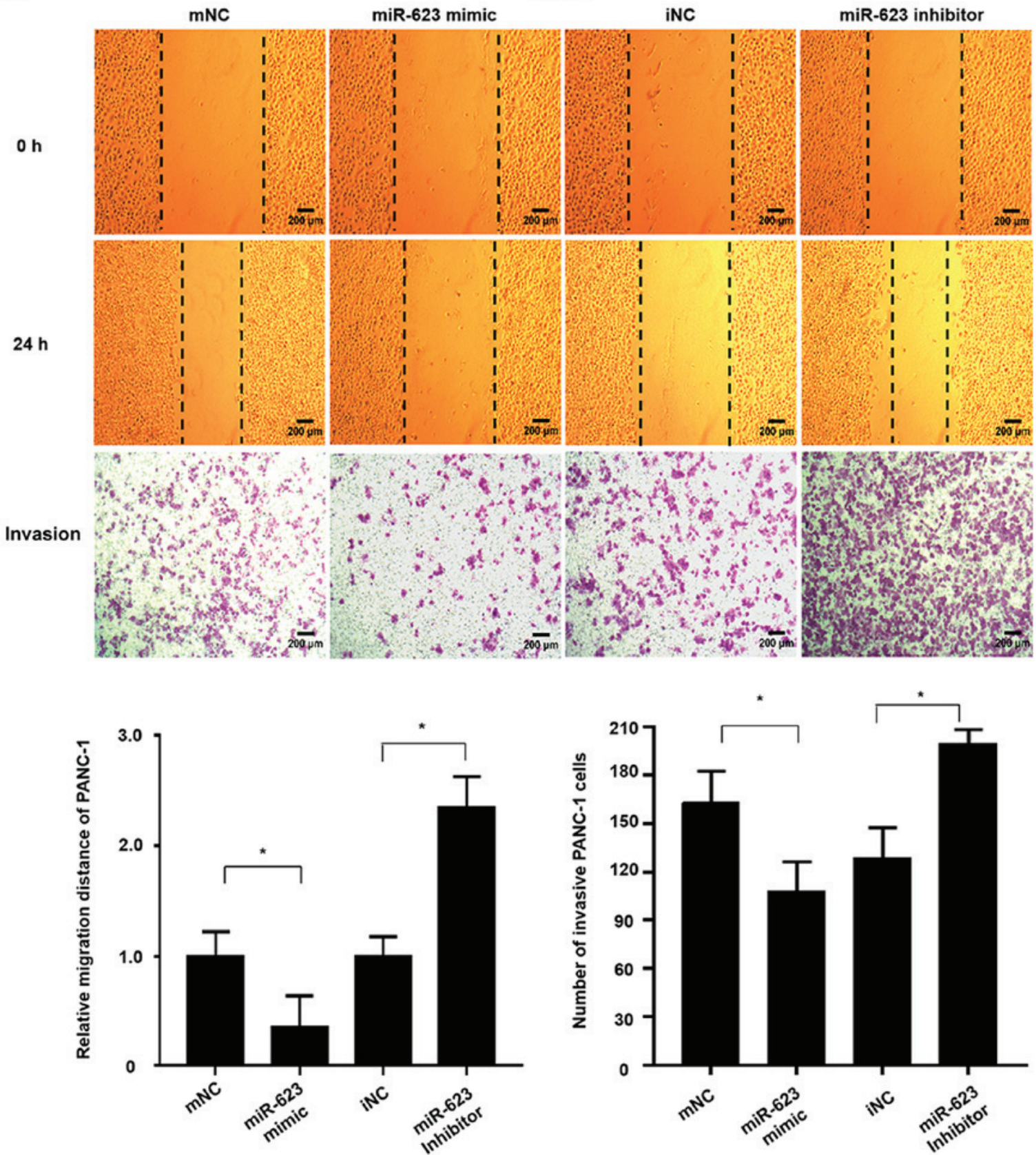

Figure 3. hsa-miR-623 inhibits the migration and invasion of PANC-1 and BXPC-3 cells. Cells were transiently transfected with $50 \mathrm{nmol} / \mathrm{m}$ hsa-miR-623 mimic, mNC, hsa-miR-623 inhibitor or iNC. A wound-healing assay was performed to investigate the migratory characteristics of the cells, and a Transwell assay was used to investigate the invasive characteristics. The hsa-miR-623 mimic inhibited, whereas the hsa-miR-623 inhibitor markedly enhanced the migration and invasion of (A) PANC-1 cells. The data are presented as the mean \pm standard deviation $(\mathrm{n}=3)$. Magnification, $\mathrm{x} 40$. " $\mathrm{P}<0.05$. miR, microRNA; $\mathrm{mNC}$, mimic negative control; iNC, inhibitor negative control.

for 6,12 and $24 \mathrm{~h}$, and E-cadherin was decreased at 12 and $24 \mathrm{~h}$ (Fig. 5C). As the ERK signaling pathway is commonly involved in mechanisms underlying IL-8 function, and exerts pro-tumor activities, western blot assays were subsequently performed to investigate whether hsa-miR-623 could suppress the phosphorylation of ERK. As hypothesized, the transfection of hsa-miR-623 mimic not only decreased ERK phosphorylation, but also decreased IL-8-induced TWIST1 and SNAI1 expression, and reversed the downregulation of E-cadherin. By contrast, transfection of the hsa-miR-623 inhibitor exhibited the opposite results (Fig. 5D). As indicated by the phalloidin staining (Fig. 5E), the morphology of the miR-623 mimic-transfected PANC-1 and BXPC-3 cells displayed relatively tight cell-cell adhesion, whereas miR-623 inhibitor-transfected cells exhibited many new spike-like filopodia at their edges, indicating a spindle-like and fibroblastic phenotype. These results suggest that upregulation of miR-623 results in inhibition of the epithelial-mesenchymal transition (EMT). Furthermore, the hsa-miR-623 mimic attenuated IL-8-induced migration and invasion of PANC-1 and BXPC-3 cells, whereas miR-623 inhibition resulted in more aggressive features (Fig. 6).

hsa-miR-623 inhibits pancreatic cancer cell metastasis in vivo. A xenograft nude mouse model was established to 
B

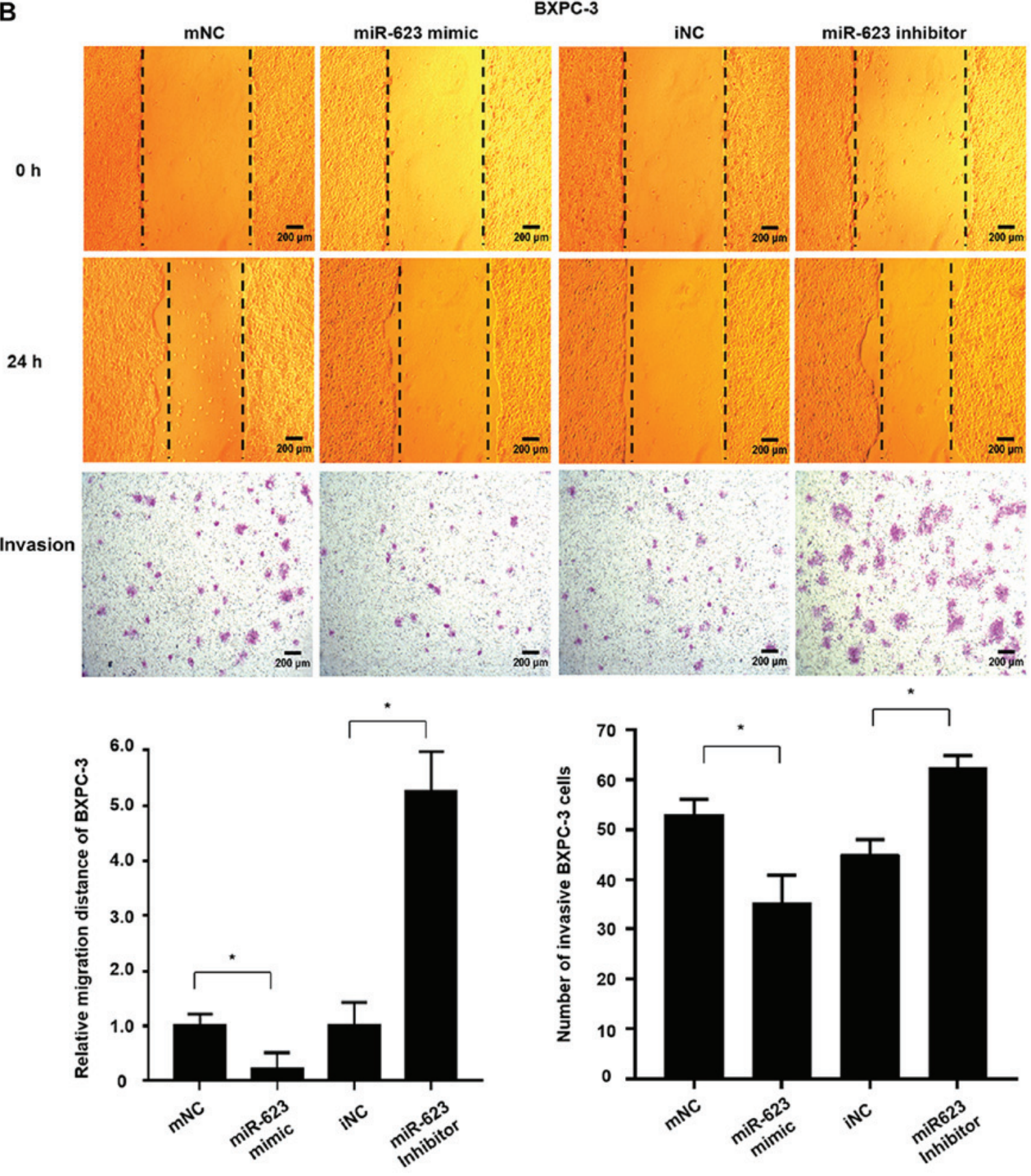

Figure 3. Continued. hsa-miR-623 inhibits the migration and invasion of PANC-1 and BXPC-3 cells. Cells were transiently transfected with $50 \mathrm{nmol} / \mathrm{m}$ hsa-miR-623 mimic, mNC, hsa-miR-623 inhibitor or iNC. A wound-healing assay was performed to investigate the migratory characteristics of the cells, and a Transwell assay was used to investigate the invasive characteristics. The hsa-miR-623 mimic inhibited, whereas the hsa-miR-623 inhibitor markedly enhanced the migration and invasion of (B) BXPC-3 cells. The data are presented as the mean \pm standard deviation ( $\mathrm{n}=3$ ). Magnification, $\mathrm{x} 40 .{ }^{*} \mathrm{P}<0.05$. miR, microRNA; $\mathrm{mNC}$, mimic negative control; iNC, inhibitor negative control.

further investigate whether hsa-miR-623 could suppress distant metastasis of PDAC in vivo. The transfection efficiency evaluated by qPCR demonstrated that Lenti-miR-623-GFP significantly increased the expression of miR-623 compared with NC transfection (Fig. 7A). The transfected PANC-1 cells were injected into the tail vein of nude mice to mimic the spread of cancer cells throughout the blood. All animals survived until the end time point of the experiment. As presented in Fig. 7B and C, heightened metastasis was observed in mice injected with rIL-8; however, when hsa-miR-623 was overexpressed in PANC-1 cells, metastasis was markedly inhibited in mice with or without IL-8 treatment. Compared with that in the control mice, the mean fluorescence density of lungs and livers in the hsa-miR-623 overexpression groups was markedly lower $(\mathrm{P}<0.05)$, indicating decreased metastasis of cancer cells. In addition, the number of lung and liver metastasis nodules was also significantly decreased in the hsa-miR-623-overexpression group compared with those in the control group (Fig. 7C and D). Furthermore, the expression of MMP1, TWIST1 and SNAI1 protein in liver metastatic nodules in the hsa-miR-623 overexpression group was also significantly decreased (Fig. 7E). These results were consistent with the in vitro results, indicating that hsa-miR-623 attenuates IL-8-induced PDAC metastasis in vitro and in vivo. 
A

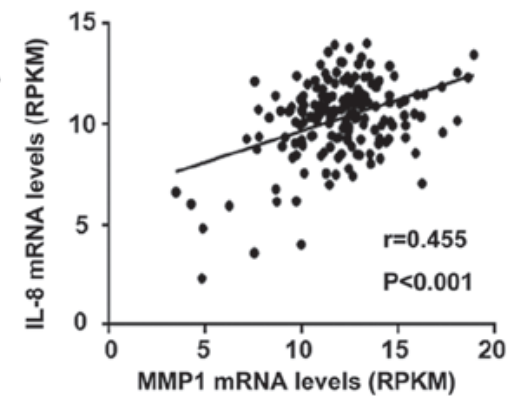

D

GSE28735

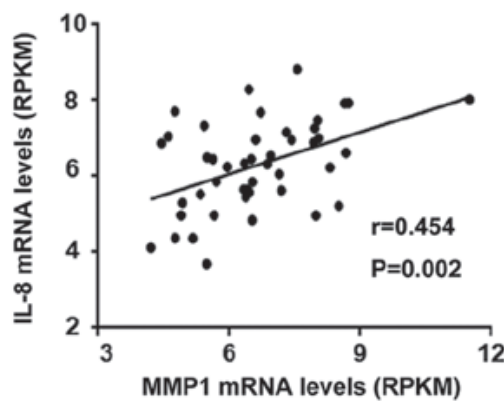

B
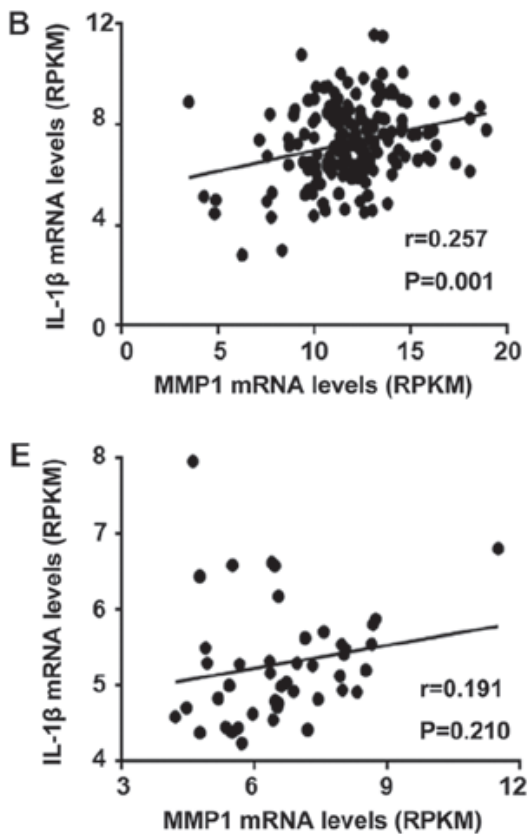

C
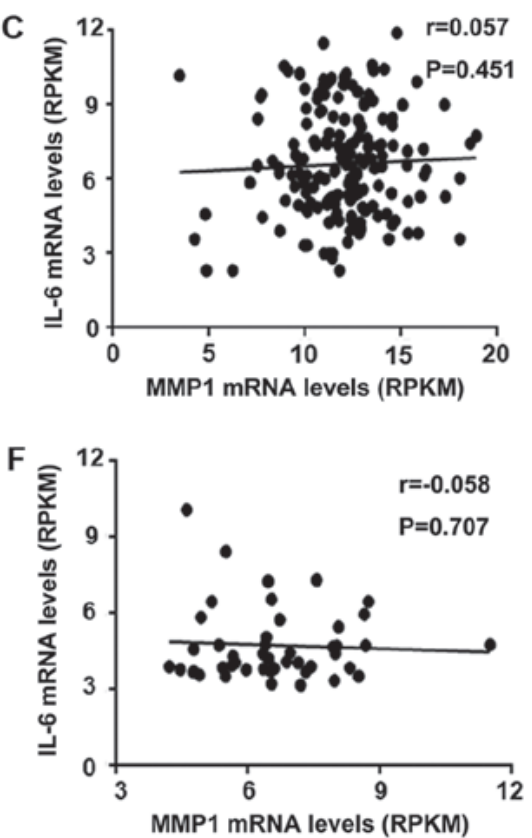

Figure 4. mRNA expression levels of MMP1 and inflammatory factors in PDAC tissues were obtained from TCGA and GEO databases. In the data from TCGA, the level of MMP1 was positively correlated with the mRNA levels of (A) IL-8 and (B) IL-1 $\beta$ in PDAC tissues. (C) No significant correlation was detected between MMP1 and IL-6 expression. In the GEO dataset GSE28735, (D) IL-8 mRNA levels exhibited a positive correlation with MMP1, whereas (E) IL-1 $\beta$ and (F) IL-6 revealed no significant correlation with MMP1 expression. MMP1, matrix metalloproteinase-1; PDAC, pancreatic ductal adenocarcinoma; TCGA, The Cancer Genome Atlas; GEO, Gene Expression Omnibus; IL, interleukin; RPKM, Reads Per Kilobase Million.

\section{Discussion}

The overexpression of MMP1 is associated with a poor prognosis in pancreatic and breast cancer, and is a novel biomarker for monitoring hepatocellular carcinoma in patients who have received liver transplants (32). MMP1 is a remarkable MMP family member due to its function as an agonist of protease-activated receptor 1 (PAR1) (33), which is involved in a plethora of pathophysiological processes, such as arterial stenosis and atherosclerosis $(34,35)$. In cancer, MMP1 activates PAR1 and exerts several pro-tumor functions, including i) promoting tumor metastasis via transendothelial migration by exerting enhanced endothelial permeability $(36,37)$,ii) inducing protein activation to promote endothelial cell angiogenesis (38), and iii) being involved with the association between perineural invasion and post-operative relapse in patients with PDAC. Our previous study demonstrated that MMP1 mediates pancreatic cancer cell perineural invasion in the early stages of disease via the MMP1/PAR1/substance P/neurokinin 1 receptor paracrine loop (39). However, clinical trials using MMP inhibitors combined with gemcitabine were unsuccessful in patients with advanced pancreatic cancer $(40,41)$. Therefore, exploring the mechanism of MMP1 and selective MMP1 inhibitors rather than targeting all MMPs may be effective for the treatment of cancer in the early stage of disease.

Ras mutations are detected in $>90 \%$ of pancreatic cancer cases at a relatively early stage. Mutated Ras results in the continuous activation of the mitogen-activated protein kinase (MAPK) pathway (ERK, JNK and p38 MAPK) and regulates the transcription of transcription factor AP-1-mediated genes. A previous study demonstrated that constitutive expression of MMP1 in human pancreatic cancer cell lines was mediated by activation of the JNK/AP-1 or ERK/AP-1 pathways, which could be suppressed by a specific inhibitor of either JNK or MAPK kinase (42). In addition to constitutive expression, MMP1 can be induced by cytokines that activate the MAPK pathway, which has been well studied in rheumatoid arthritis and joint damage (43). Similar to inflammatory diseases, MMP1 expression in neoplastic cells is also inducible, and exerts a pivotal function in the progression of cancer (44). Taking into consideration that MMP1 expression is affected by several factors, it is conceivable that modulators that inhibit MMP1 expression at the transcriptional and post-transcriptional levels will exert potent anti-invasive effects on pancreatic cancer cells.

Mature miRNAs recognize the 3'-UTR of their target mRNA, subsequently forming miRNA/mRNA/protein complexes that incorporate into the RNA-induced silencing complex, which ultimately results in mRNA cleavage (45). The present study screened miRNAs that potentially modulate MMP1 and analyzed the correlation between miRNAs and MMP1 mRNA in PDAC tissues, discovering that only the expression of hsa-miR-623 exhibited a negative correlation with MMP1 mRNA expression. By contrast, other miRNAs that have been reported to target MMP1 in different types of non-tumor diseases exhibited no correlation with MMP1 mRNA expression. These findings may be explained by the heterogeneity of pancreatic cancer cells. An RNA EMSA was subsequently used to demonstrate the direct and specific interaction between hsa-miRNA-623 and its target within the 3'-UTR of MMP1, and a luciferase reporter assay confirmed the downregulation of MMP1 in response to hsa-miR-623 targeting its 3'-UTR. miRNAs also regulate gene expression indirectly, by weaving a complex regulation network in cancer, as supported by the western blotting results following cell transfection. The upregulation of hsa-miRNA-623 not only decreased MMP1 protein expression, but also 
A

PANC-1

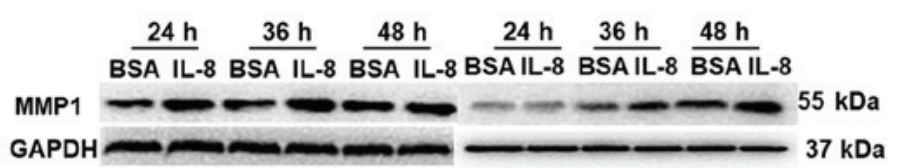

\section{B}

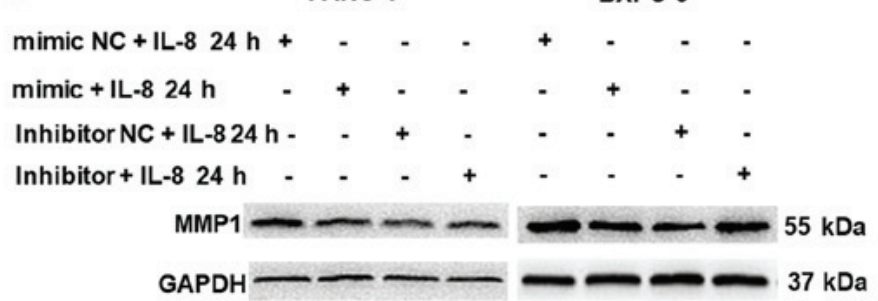

C

\begin{tabular}{lllll} 
& PANC-1 & \multicolumn{2}{c}{ BXPC-3 } \\
\hline $\mathrm{h}$ & $\underline{12 \mathrm{~h}} \quad \underline{24 \mathrm{~h}} \quad \underline{6 \mathrm{~h}} \quad \underline{12 \mathrm{~h}} \quad \underline{24 \mathrm{~h}}$
\end{tabular} BSA IL-8 BSA IL-8 $\overline{\text { BSA IL-8 BSA IL-8 BSA IL-8 BSA IL-8 }}$

$\mathrm{E}-\mathrm{cad} \longrightarrow \mathrm{B}-\mathrm{m}=\mathrm{w}-120 \mathrm{kDa}$ TWIST1 - - - - - - - - $30 \mathrm{kDa}$

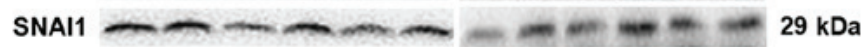
GAPDH

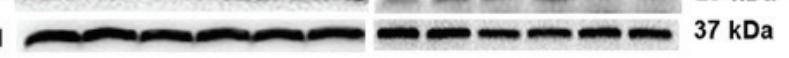

D

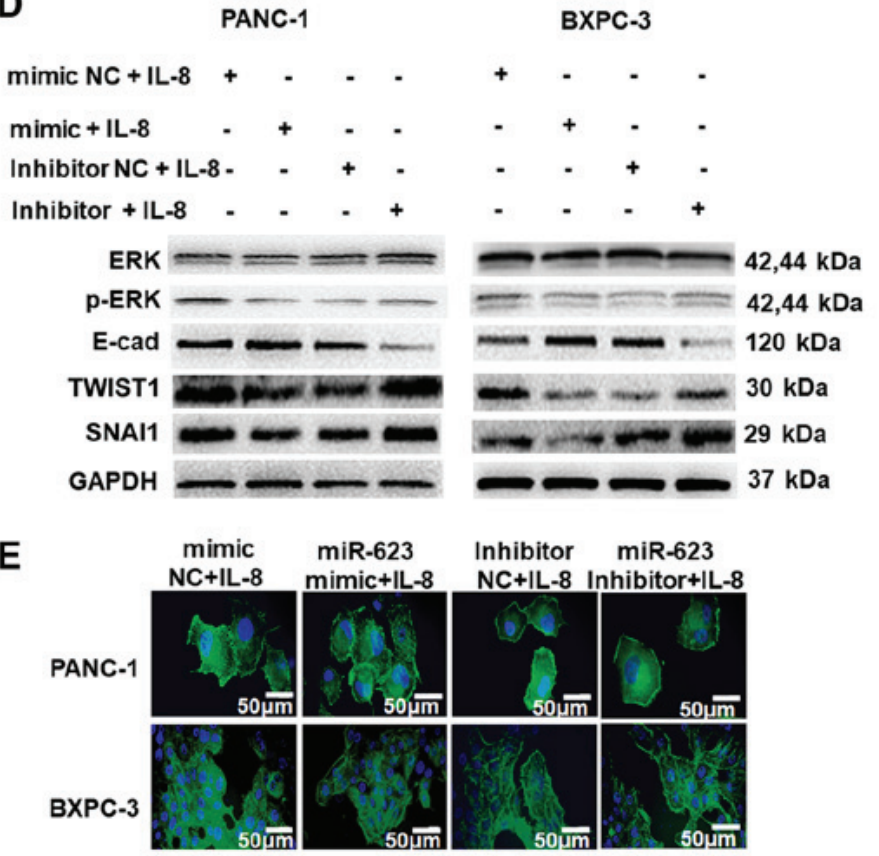

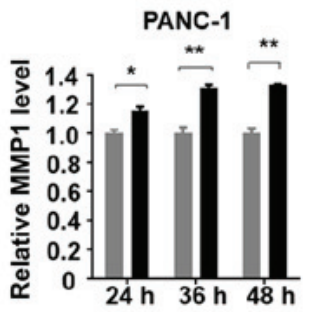
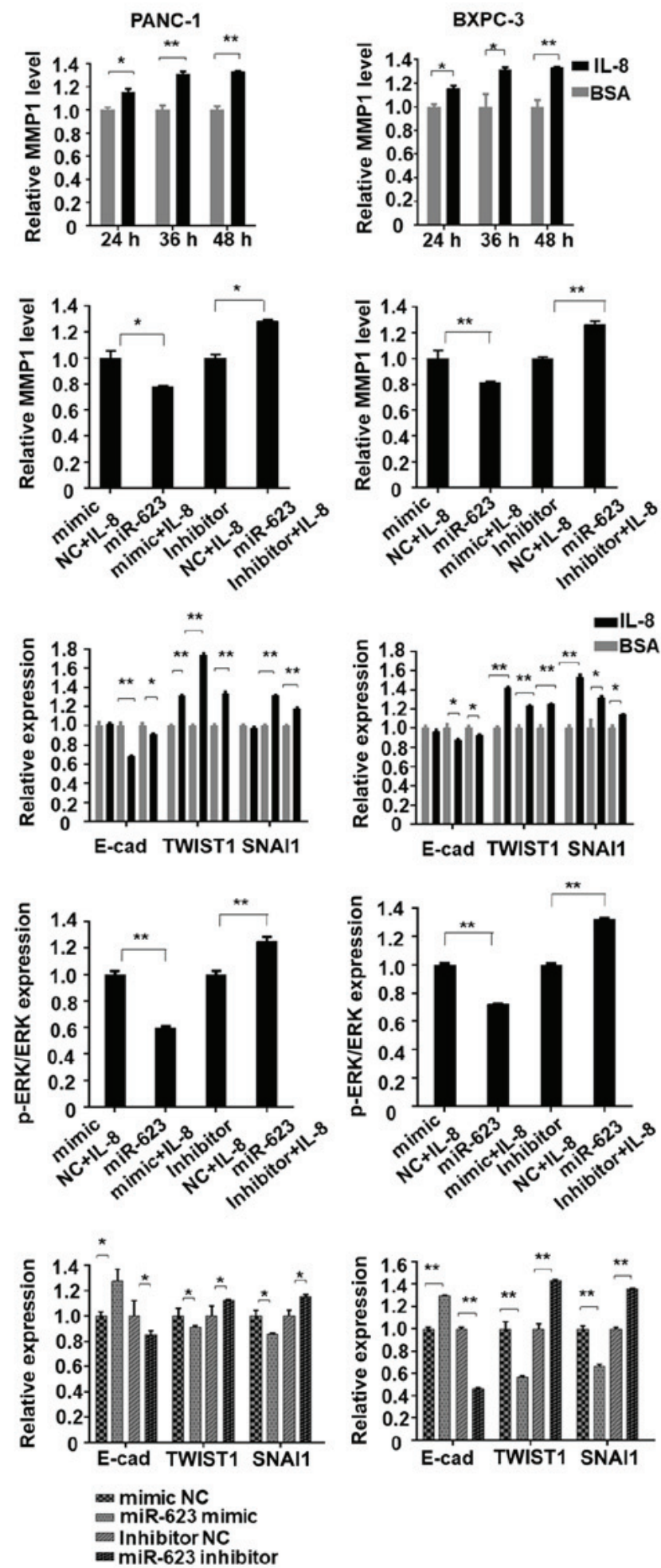

Figure 5. hsa-miR-623 attenuates IL-8-induced MMP1 expression and the epithelial-mesenchymal transition in pancreatic cancer cells. (A) Treatment with recombinant human IL-8 increased the levels of MMP1, whereas (B) hsa-miR-623 inhibitor led to the upregulation of MMP1 levels. (C) Recombinant IL-8 treatment increased the expression of TWIST1 and SNAI1, and decreased the expression of E-cad in PANC-1 and BXPC-3 cells. (D) The hsa-miR-623 mimic inhibited the IL-8-induced upregulation of the epithelial-mesenchymal transition by suppressing ERK phosphorylation. (E) Cells were stained with phalloidin (green) and counterstained with DAPI (blue). Magnification, $\mathrm{x} 400 .{ }^{*} \mathrm{P}<0.05,{ }^{* *} \mathrm{P}<0.001$. IL, interleukin; MMP1, matrix metalloproteinase-1; miR, microRNA; E-cad, E-cadherin; TWIST1, Twist-related protein 1; SNAI1, zinc finger protein SNAI1; p-ERK, phosphorylated ERK; NC, negative control.

suppressed the phosphorylation of ERK, which is part of one of the essential pathways for AP1-dependent MMP1 transcription (42). Although the exact mechanism whereby hsa-miRNA-623 inhibits the phosphorylation of ERK requires further investigation, the results of the present study indicate that hsa-miR-623 may downregulate MMP1 expression directly by post-transcriptional degradation and indirectly by transcriptional inhibition.

A previous study reported that hsa-miR-623 inhibits the proliferation and metastasis of human lung adenocarcinoma 

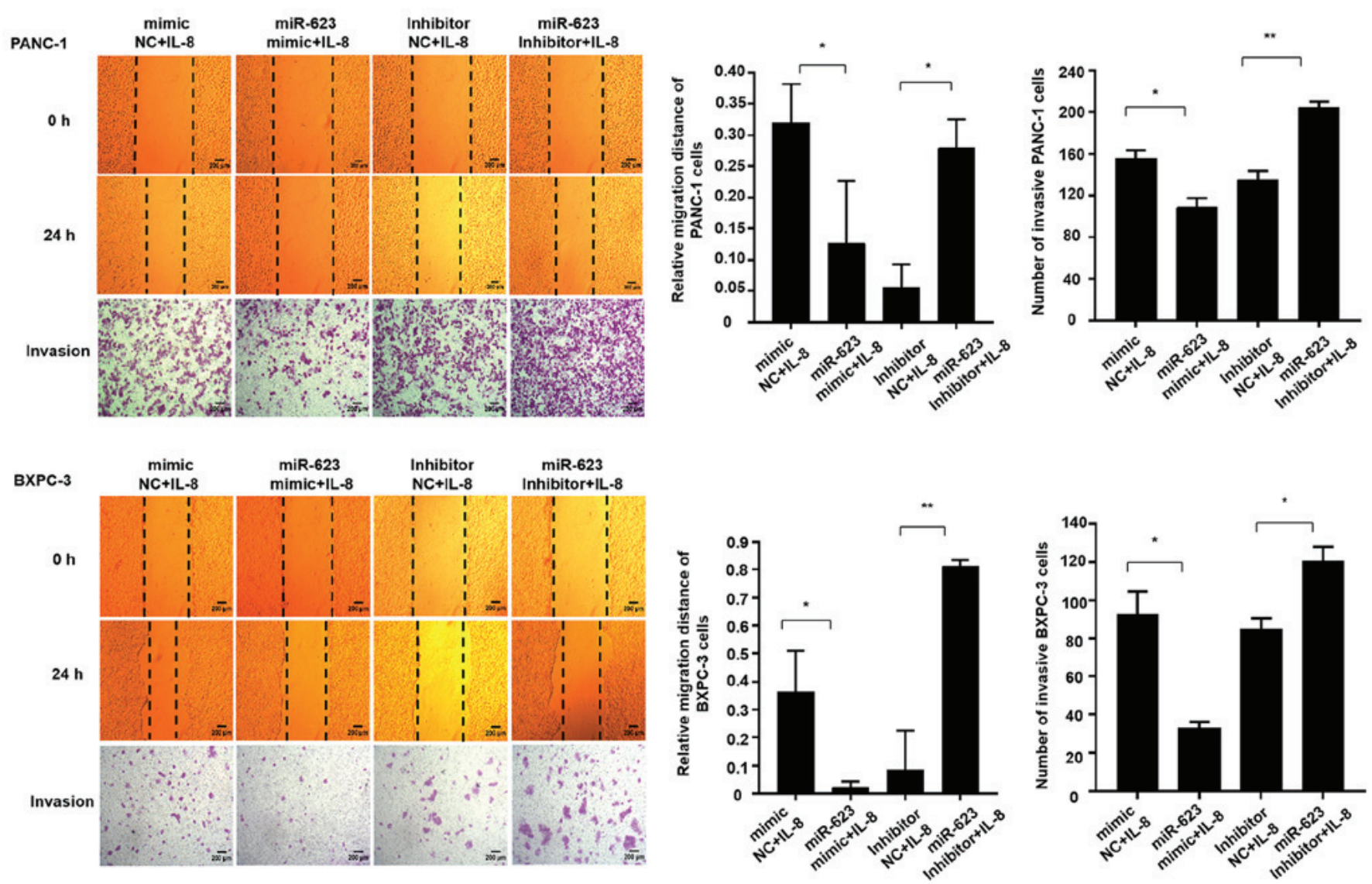

Figure 6. Migration and invasion of pancreatic cancer cells treated with IL-8. hsa-miR-623 mimic inhibited, whereas its inhibitor enhanced, the pro-migratory and pro-invasive function of IL-8 in pancreatic cancer PANC-1 and BXP-3 cells. The data are presented as the mean \pm standard deviation ( $\mathrm{n}=3$ ). ${ }^{*} \mathrm{P}<0.05$, ${ }^{* *} \mathrm{P}<0.001$. Magnification, $\mathrm{x} 40$. IL, interleukin; miR, microRNA; NC, negative control.

by targeting X-ray repair cross-complementing protein 5 (46). However, overexpression of hsa-miR-623 exhibited no apparent influence on PANC-1 or BXPC-3 cell proliferation (data not shown); therefore, in the present study, the primary focus was on the anti-metastatic effects of hsa-miR-623 on PDAC cells. The EMT is a crucial driver of cancer cell migration and invasion, which is regulated by various molecular mechanisms, including pro-inflammatory factors and MMP activity (47). SNAI1 and TWIST1 are two important transcription factors, referred to as EMT transcription factors (EMT-TFs), which exert pivotal functions in the process of EMT (48). Exogenous factors that induce the expression of SNAI1 or TWIST1 (IL-8 and IL-6) and endogenous regulators (miRNAs) contribute to cancer metastasis $(49,50)$. The results of the present study demonstrated that hsa-miR-623 is involved in this cellular/factor network: First, hsa-miR-623 downregulated MMP1 by targeting the 3'-UTR. Secondly, IL-8 induced the expression of MMP1, SNAI1 and TWIST1, whereas hsa-miR-623 attenuated the induction effect of IL-8 via the underlying mechanism of ERK phosphorylation suppression. Since SNAI1 and TWIST1 are EMT-TFs that inhibit the expression of E-cadherin, one of the most important EMT-regulating genes, through E-boxes in the promoter region (51), hsa-miR-623 upregulated E-cadherin expression and inhibited EMT. Finally, hsa-miR-623 inhibited PANC-1 and BXPC-3 cell invasion in vitro and in vivo.

IL- 8 has been reported to induce MMPs, particularly MMP2 and 9 , which are overexpressed in various types of cancer, whereas studies investigating IL-8 and MMP1 are limited. It appears that IL- 8 and MMP1 are mutual promoting factors, and IL-8 induces MMP1 expression via IL-8/androgen receptor signaling in bladder cancer cells (8); in addition, IL-8/C-X-C chemokine receptor types 1 and 2 (CXCR1/2) activates ERK1/2 (52) and may upregulate MMP1 expression via ERK/AP-1 signaling. On the other hand, in ovarian cancer cells, MMP1 induces IL-8 production in a PAR1-dependent manner; subsequently, secreted IL-8 activates CXCR $1 / 2$ receptors on endothelial cells, thereby causing angiogenesis and metastasis (53).

A database analysis verified a positive correlation between IL-8 and MMP1, and a negative correlation between MMP1 and the expression of hsa-miR-623 in PDAC tissues. Therefore, the MMP1-PAR1/IL-8-CXCR1/2 paracrine pathway may be therapeutically relevant for the treatment of pancreatic cancer $(22,54)$. As a direct suppressor of MMP1, hsa-miR-623 may consequently block this paracrine loop and therefore exert antitumor activity by modulating multiple steps.

In summary, the present study demonstrated that hsa-miR-623 binds to a specific sequence located within the 3'-UTR of MMP1 and suppresses MMP1 expression. In addition, hsa-miR-623 suppressed the IL-8-induced expression of MMP1 and several EMT functional proteins, consequently inhibiting pancreatic cancer metastasis. These results may provide insights pertaining to a novel epigenetic mechanism for regulating the expression of the MMP1 gene and suggest 
A

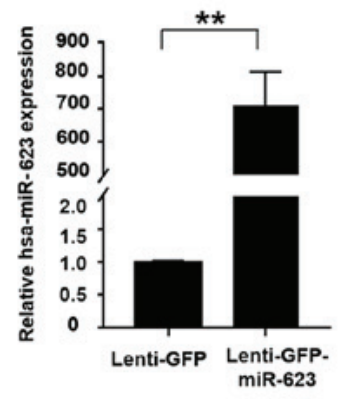

B
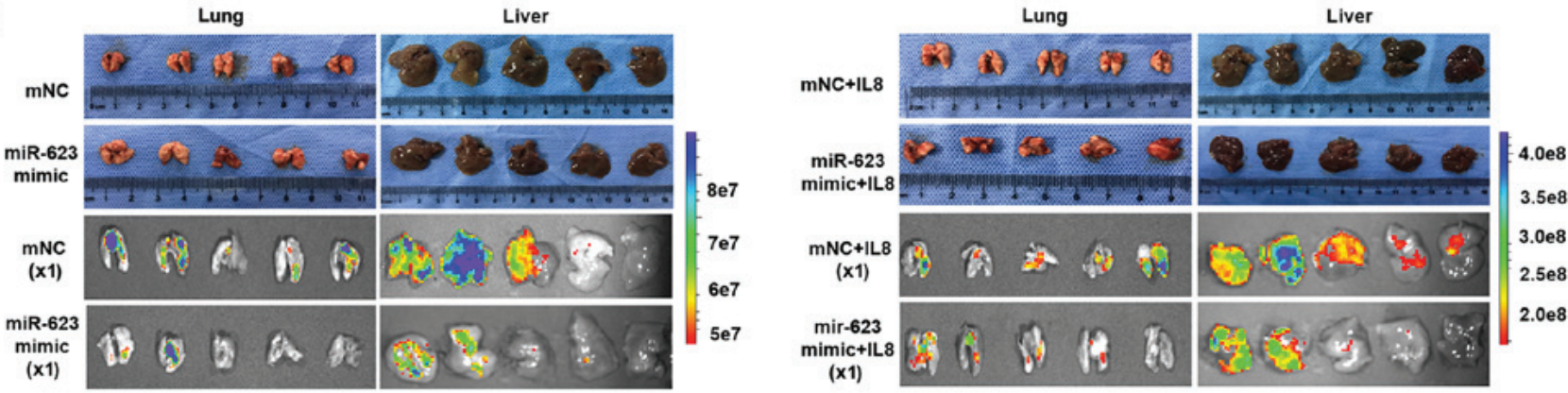

C
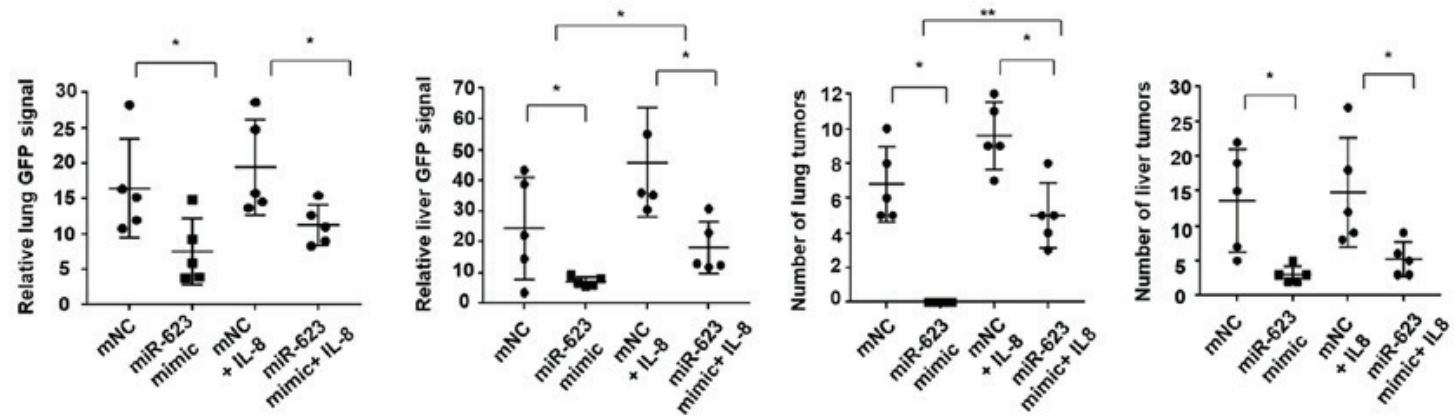

D

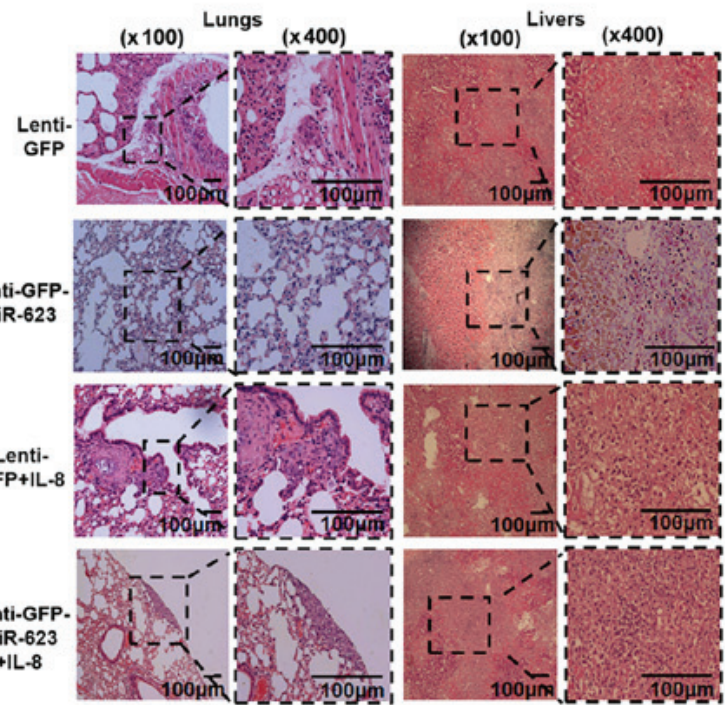

E

H\&E ( $\times 400) \quad$ MMP1 $(\times 400) \quad$ SNAI1 $(\times 400)$ TWIST1 $(\times 400)$ E-cad $(\times 400)$

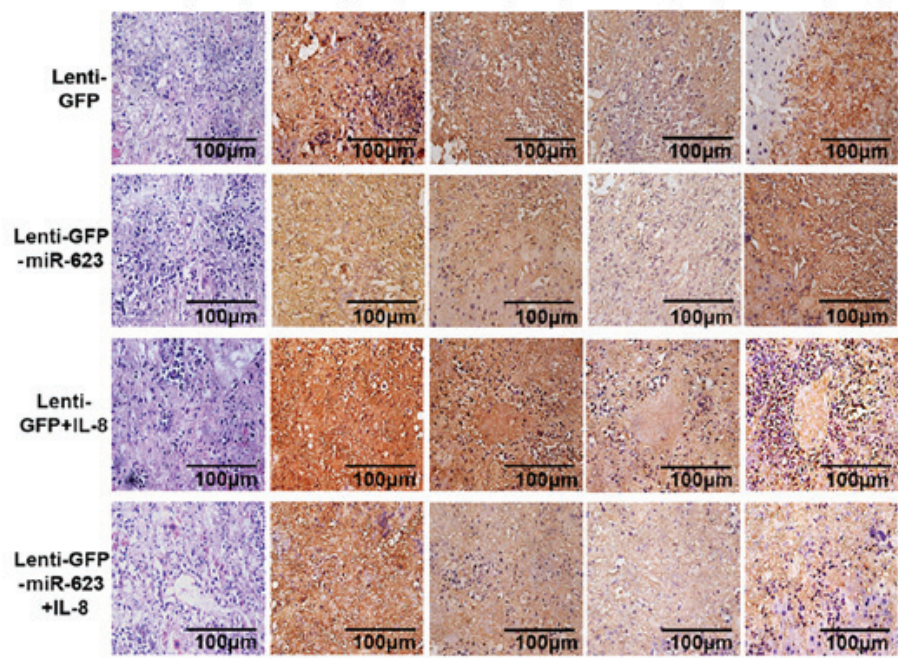

Figure 7. hsa-miR-623 inhibits pancreatic cancer cell metastasis in vivo. A total of $2 \times 10^{7}$ PANC-1 cells pre-transfected with lenti-GFP or lenti-miR-623-GFP were injected into the tail veins of 5-week-old nude mice. Recombinant human IL-8 was administered via intraperitoneal injection at a dosage of $100 \mathrm{ng} / \mathrm{animal}$ once a week for a period of 5 weeks. (A) The transfection efficiency was evaluated by reverse transcription-quantitative PCR, and the results revealed that the lenti-miR-623-GFP increased the expression of miR-623 to 706.43 \pm 73.17 times that of the lenti-GFP transfection $(\mathrm{P}<0.001)$. Increased levels of distant metastasis were observed in mice that had been treated with IL-8. The livers and lungs were isolated, and metastatic lesions were evaluated by (B-C) fluorescence scanning and (D) tissue section HE staining. Mice injected with PANC-1 lenti-miR-623-GFP cells exhibited decreased liver and lung metastasis compared with those injected with PANC-1 lenti-GFP cells with or without IL-8 treatment. (E) Immunochemical staining of tumor sections. The expression of MMP1, TWIST1 and SNAI1 protein in liver metastatic nodules from the hsa-miR-623-overexpression group was decreased, and the E-cad protein expression increased. The data are presented as the mean \pm standard deviation $(\mathrm{n}=3) .{ }^{*} \mathrm{P}<0.05,{ }^{* *} \mathrm{P}<0.001$. miR, microRNA; GFP, green fluorescent protein; IL, interleukin; HE, hematoxylin and eosin; MMP1, matrix metalloproteinase-1; TWIST1, Twist-related protein 1; SNAI1, zinc finger protein SNAI1; E-cad, E-cadherin; $\mathrm{mNC}$, mimic negative control. 
that hsa-miR-623 may be a novel adjuvant therapeutic target to prevent pancreatic cancer metastasis.

\section{Acknowledgements}

Not applicable.

\section{Funding}

This study was supported by the National Natural Science Foundation of China (grant nos. 81872348 and 81502503) and the Natural Science Foundation of Guangdong Province, China (grant no. 2016A030310191).

\section{Availability of data and materials}

The datasets used and/or analyzed during the current study are available from the corresponding author on reasonable request.

\section{Authors' contributions}

YuC, SP and $\mathrm{CH}$ carried out the experimental work, data collection and interpretation. HC and YL participated in the animal experiments. HS, YS and LZ participated in the study design and drafted the manuscript. YiC carried out data collection and analysis. All authors read and approved the final manuscript.

\section{Ethics approval and consent to participate}

All animal experiments were approved by the Animal Care and Use Committee of Sun Yat-Sen University, Guangzhou, China (approval no. 180604).

\section{Patient consent for publication}

Not applicable.

\section{Competing interests}

The authors declare that they have no competing interests.

\section{References}

1. Kamisawa T, Wood LD, Itoi T and Takaori K: Pancreatic cancer. Lancet 388: 73-85, 2016.

2. Kamisawa T, Isawa T, Koike M, Tsuruta $\mathrm{K}$ and Okamoto A: Hematogenous metastases of pancreatic ductal carcinoma. Pancreas 11: 345-349, 1995.

3. Conroy T, Bachet JB, Ayav A, Huguet F, Lambert A, Caramella C, Maréchal R, Van Laethem JL and Ducreux M: Current standards and new innovative approaches for treatment of pancreatic cancer. Eur J Cancer 57: 10-22, 2016.

4. Bonnans C, Chou J and Werb Z: Remodelling the extracellular matrix in development and disease. Nat Rev Mol Cell Biol 15: 786-801, 2014

5. Shen TC, Chang WS, Tsai CW, Chao CY, Lin YT, Hsiao CL, Hsu CL, Chen WC, Hsia TC and Bau DT: The contribution of matrix metalloproteinase-1 promoter genotypes in Taiwan lung cancer risk. Anticancer Res 38: 253-257, 2018.

6. Padala C, Tupurani MA, Puranam K, Gantala S, Shyamala N, Kondapalli MS, Gundapaneni KK, Mudigonda S, Galimudi RK, Kupsal K, et al: Synergistic effect of collagenase-1 (MMP1), stromelysin-1 (MMP3) and gelatinase-B (MMP9) gene polymorphisms in breast cancer. PLoS One 12: e0184448, 2017.
7. Hu W, Ye Y, Yin Y, Sang P, Li L, Wang J, Wan W, Li R, Bai X, Xie Y, et al: Association of matrix metalloprotease 1, 3, and 12 polymorphisms with rheumatic heart disease in a Chinese Han population. BMC Med Genet 19: 27, 2018.

8. Ou Z, Wang Y, Liu L, Li L, Yeh S, Qi L and Chang C: Tumor microenvironment $\mathrm{B}$ cells increase bladder cancer metastasis via modulation of the IL-8/androgen receptor (AR)/MMPs signals. Oncotarget 6: 26065-26078, 2015.

9. Yang G, Im HJ and Wang JH: Repetitive mechanical stretching modulates IL-1beta induced COX-2, MMP-1 expression, and PGE2 production in human patellar tendon fibroblasts. Gene 363: 166-172, 2005.

10. Li R, Hebert JD, Lee TA, Xing H, Boussommier-Calleja A, HynesRO,LauffenburgerDA and KammRD:Macrophage-secreted TNF $\alpha$ and TGF $\beta 1$ influence migration speed and persistence of cancer cells in 3D tissue culture via independent pathways. Cancer Res 77: 279-290, 2017.

11. Croce CM: Oncogenes and cancer. N Engl J Med 358: 502-511, 2008.

12. Rozario $\mathrm{T}$ and DeSimone DW: The extracellular matrix in development and morphogenesis: A dynamic view. Dev Biol 341: 126-140, 2010.

13. Marchesi F, Regazzo G, Palombi F, Terrenato I, Sacconi A, Spagnuolo M, Donzelli S, Marino M, Ercolani C, Di Benedetto A, et al: Serum miR-22 as potential non-invasive predictor of poor clinical outcome in newly diagnosed, uniformly treated patients with diffuse large B-cell lymphoma: An explorative pilot study. J Exp Clin Cancer Res 37: 95, 2018.

14. Samir N, Matboli M, El-Tayeb H, El-Tawdi A, Hassan MK, Waly A, El-Akkad HAE, Ramadan MG, Al-Belkini TN, El-Khamisy S, et al: Competing endogenous RNA network crosstalk reveals novel molecular markers in colorectal cancer. J Cell Biochem 119: 6869-6881, 2018.

15. Frampton AE, Castellano L, Colombo T, Giovannetti E, Krell J, Jacob J, Pellegrino L, Roca-Alonso L, Funel N, Gall TM, et al: Integrated molecular analysis to investigate the role of microRNAs in pancreatic tumour growth and progression. Lancet 385 (Suppl 1): S37, 2015.

16. Zhang Y, Lin X, Zhang L, Hong W and Zeng K: MicroRNA-222 regulates the viability of fibroblasts in hypertrophic scars via matrix metalloproteinase 1. Exp Ther Med 15: 1803-1808, 2018.

17. Kim KH, Jung JY, Son ED, Shin DW, Noh M and Lee TR: miR-526b targets 3' UTR of MMP1 mRNA. Exp Mol Med 47: e178, 2015.

18. Wang Y, Pang X, Wu J, Jin L, Yu Y, Gobin R and Yu J: MicroRNA hsa-let-7b suppresses the odonto/osteogenic differentiation capacity of stem cells from apical papilla by targeting MMP1. J Cell Biochem 119: 6545-6554, 2018.

19. Zhou B, Zhu H, Luo H, Gao S, Dai X, Li Y and Zuo X: MicroRNA-202-3p regulates scleroderma fibrosis by targeting matrix metalloproteinase 1. Biomed Pharmacother 87: 412-418, 2017.

20. Yu D, Green B, Marrone A, Guo Y, Kadlubar S, Lin D, Fuscoe J, Pogribny I and Ning B: Suppression of CYP2C9 by microRNA hsa-miR-128-3p in human liver cells and association with hepatocellular carcinoma. Sci Rep 5: 8534, 2015.

21. Chen Y, Zeng L, Wang Y, Tolleson WH, Knox B, Chen S, Ren Z, Guo L, Mei N, Qian F, et al: The expression, induction and pharmacological activity of CYP1A2 are post-transcriptionally regulated by microRNA hsa-miR-132-5p. Biochem Pharmacol 145: 178-191, 2017.

22. Chen L, Fan J, Chen H, Meng Z, Chen Z, Wang P and Liu L: The IL-8/CXCR1 axis is associated with cancer stem cell-like properties and correlates with clinical prognosis in human pancreatic cancer cases. Sci Rep 4: 5911, 2014.

23. Jaillon S, Peri G, Delneste Y, Frémaux I, Doni A, Moalli F, Garlanda C, Romani L, Gascan H, Bellocchio S, et al: The humoral pattern recognition receptor PTX3 is stored in neutrophil granules and localizes in extracellular traps. J Exp Med 204: 793-804, 2007.

24. Livak KJ and Schmittgen TD: Analysis of relative gene expression data using real-time quantitative PCR and the $2(-\Delta \Delta C(T))$ method. Methods 25: 402-408, 2001.

25. Zhang G, Schetter A, He P, Funamizu N, Gaedcke J, Ghadimi BM, Ried T, Hassan R, Yfantis HG, Lee DH, et al: DPEP1 inhibits tumor cell invasiveness, enhances chemosensitivity and predicts clinical outcome in pancreatic ductal adenocarcinoma. PLoS One 7: e31507, 2012. 
26. Donahue TR, Tran LM, Hill R, Li Y, Kovochich A, Calvopina JH, Patel SG, Wu N, Hindoyan A, Farrell JJ, et al: Integrative survival-based molecular profiling of human pancreatic cancer. Clin Cancer Res 18: 1352-1363, 2012.

27. Zeng L, Chen Y, Wang Y, Yu LR, Knox B, Chen J, Shi T, Chen S, Ren Z, Guo L, et al: MicroRNA hsa-miR-370-3p suppresses the expression and induction of CYP2D6 by facilitating mRNA degradation. Biochem Pharmacol 140: 139-149, 2017.

28. Wang Y, Yu D, Tolleson WH, Yu LR, Green B, Zeng L, Chen Y, Chen S, Ren Z, Guo L, et al: A systematic evaluation of microRNAs in regulating human hepatic CYP2E1. Biochem Pharmacol 138: 174-184, 2017.

29. Jin Y, Yu D, Tolleson WH, Knox B, Wang Y, Chen S, Ren Z, Deng $\mathrm{H}$, Guo $\mathrm{Y}$ and Ning B: MicroRNA hsa-miR-25-3p suppresses the expression and drug induction of CYP2B 6 in human hepatocytes. Biochem Pharmacol 113: 88-96, 2016.

30. Trenkmann M, Brock M, Gay RE, Michel BA, Gay S and Huber LC: Tumor necrosis factor $\alpha$-induced microRNA-18a activates rheumatoid arthritis synovial fibroblasts through a feedback loop in NF- $\kappa$ B signaling. Arthritis Rheum 65: 916-927, 2012.

31. Yoon HY, Lee EG, Lee H, Cho IJ, Choi YJ, Sung MS, Yoo HG and Yoo WH: Kaempferol inhibits IL-1 $\beta$-induced proliferation of rheumatoid arthritis synovial fibroblasts and the production of COX-2, PGE2 and MMPs. Int J Mol Med 32: 971-977, 2013.

32. Sánchez-Lorencio MI, Saenz L, Ramirez P, Villalba-López F, de la Orden V, Mediero-Valeros B, Revilla Nuin B, Gonzalez MR, Cascales-Campos PA, Ferreras-Martínez D, et al: Matrix metalloproteinase 1 as a novel biomarker for monitoring hepatocellular carcinoma in liver transplant patients. Transplant Proc 50: 623-627, 2018.

33. Trivedi V, Boire A, Tchernychev B, Kaneider NC, Leger AJ, O'Callaghan K, Covic L and Kuliopulos A: Platelet matrix metalloprotease-1 mediates thrombogenesis by activating PAR1 at a cryptic ligand site. Cell 137: 332-343, 2009.

34. Austin KM, Nguyen N, Javid G, Covic L and Kuliopulos A: Noncanonical matrix metalloprotease-1-protease-activated receptor-1 signaling triggers vascular smooth muscle cell dedifferentiation and arterial stenosis. J Biol Chem 288: 23105-23115, 2013.

35. Rana R, Huang T, Koukos G, Fletcher EK, Turner SE, Shearer A, Gurbel PA, Rade JJ, Kimmelstiel CD, Bliden KP, et al: Noncanonical matrix metalloprotease 1-protease-activated receptor 1 signaling drives progression of atherosclerosis. Arterioscler Thromb Vasc Biol 38: 1368-1380, 2018.

36. Juncker-Jensen A, Deryugina EI, Rimann I, Zajac E, Kupriyanova TA, Engelholm LH and Quigley JP: Tumor MMP-1 activates endothelial PAR1 to facilitate vascular intravasation and metastatic dissemination. Cancer Res 73: 4196-4211, 2013

37. Goerge T, Barg A, Schnaeker EM, Poppelmann B, Shpacovitch V, Rattenholl A, Maaser C, Luger TA, Steinhoff M and Schneider SW: Tumor-derived matrix metalloproteinase-1 targets endothelial proteinase-activated receptor 1 promoting endothelial cell activation. Cancer Res 66: 7766-7774, 2006.

38. Gopal SK, Greening DW, Zhu HJ, Simpson RJ and Mathias RA: Transformed MDCK cells secrete elevated MMP1 that generates LAMA5 fragments promoting endothelial cell angiogenesis. Sci Rep 6: 28321, 2016.

39. Huang C, Li Y, Guo Y, Zhang Z, Lian G, Chen Y, Li J, Su Y, Li J, Yang K, et al: MMP1/PAR1/SP/NK1R paracrine loop modulates early perineural invasion of pancreatic cancer cells. Theranostics 8: 3074-3086, 2018.
40. Bramhall SR, Rosemurgy A, Brown PD, Bowry C and Buckels JA; Marimastat Pancreatic Cancer Study Group: Marimastat as first-line therapy for patients with unresectable pancreatic cancer: A randomized trial. J Clin Oncol 19: 3447-3455, 2001.

41. Bramhall SR, Schulz J, Nemunaitis J, Brown PD, Baillet M and Buckels JA: A double-blind placebo-controlled, randomised study comparing gemcitabine and marimastat with gemcitabine and placebo as first line therapy in patients with advanced pancreatic cancer. Br J Cancer 87: 161-167, 2002.

42. Endo H, Watanabe T, Sugioka Y, Niioka M, Inagaki Y and Okazaki I: Activation of two distinct MAPK pathways governs constitutive expression of matrix metalloproteinase-1 in human pancreatic cancer cell lines. Int J Oncol 35: 1237-1245, 2009.

43. Maksymowych WP, van der Heijde D, Allaart CF, Landewé R, Boire G, Tak PP, Gui Y, Ghahary A, Kilani R and Marotta A: $14-3-3 \eta$ is a novel mediator associated with the pathogenesis of rheumatoid arthritis and joint damage. Arthritis Res Ther 16: R99, 2014.

44. Ha NH, Park DG, Woo BH, Kim DJ, Choi JI, Park BS, Kim YD, Lee JH and Park HR: Porphyromonas gingivalis increases the invasiveness of oral cancer cells by upregulating IL- 8 and MMPs. Cytokine 86: 64-72, 2016.

45. Gregory RI, Chendrimada TP, Cooch N and Shiekhattar R: Human RISC couples microRNA biogenesis and posttranscriptional gene silencing. Cell 123: 631-640, 2005.

46. Wei S, Zhang ZY, Fu SL, Xie JG, Liu XS, Xu YJ, Zhao JP and Xiong WN: Hsa-miR-623 suppresses tumor progression in human lung adenocarcinoma. Cell Death Dis 8: e2829, 2017.

47. Nieto MA, Huang RY, Jackson RA and Thiery JP: Emt: 2016. Cell 166: 21-45, 2016.

48. Onder TT, Gupta PB, Mani SA, Yang J, Lander ES and Weinberg RA: Loss of E-cadherin promotes metastasis via multiple downstream transcriptional pathways. Cancer Res 68: 3645-3654, 2008.

49. Dominguez C, David JM and Palena C: Epithelial-mesenchymal transition and inflammation at the site of the primary tumor. Semin Cancer Biol 47: 177-184, 2017.

50. Ding XM: MicroRNAs: Regulators of cancer metastasis and epithelial-mesenchymal transition (EMT). Chin J Cancer 33: 140-147, 2014.

51. Thiery JP, Acloque H, Huang RY and Nieto MA: Epithelialmesenchymal transitions in development and disease. Cell 139: 871-890, 2009.

52. Nasser MW, Raghuwanshi SK, Grant DJ, Jala VR, Rajarathnam K and Richardson RM: Differential activation and regulation of CXCR 1 and CXCR2 by CXCL8 monomer and dimer. J Immunol 183: 3425-3432, 2009.

53. Agarwal A, Tressel SL, Kaimal R, Balla M, Lam FH, Covic L and Kuliopulos A: Identification of a metalloprotease-chemokine signaling system in the ovarian cancer microenvironment: Implications for antiangiogenic therapy. Cancer Res 70: 5880-5890, 2010.

54. Queiroz KC, Shi K, Duitman J, Aberson HL, Wilmink JW, van Noesel CJ, Richel DJ and Spek CA: Protease-activated receptor-1 drives pancreatic cancer progression and chemoresistance. Int J Cancer 135: 2294-2304, 2014.

This work is licensed under a Creative Commons Attribution-NonCommercial-NoDerivatives 4.0 International (CC BY-NC-ND 4.0) License. 